\title{
Verhandlungen der gynäkologischen Section des $X$. internationalen medicin. Congresses in Berlin vom 4. bis 9. August 1890 .
}

\author{
Bericht \\ von \\ Privatdocent Dr. Dührssen, \\ Berlin.
}

\section{Sitzung am 4. August Nachmittags.}

Vorsitzender: Dohrn (Königsberg).

I. Allgemeines Thema: Antisepsis in der Geburtshülfe.

1) Referent Galabin (London) war durch Krankheit am Erscheinen verhindert, hatte aber Thesen eingeschickt, nach welchen die Geburtshülfe ihre Erfolge in erster Linie dem Sublimate verdankt. Sublimatausspülungen der Scheide von $1: 2000$ empfiehlt Galabin auch nach normalen Geburten in der Privatpraxis.

2) Correferent v. Slavjansky (Petersburg) bespricht die Antisepsis in den Gebäranstalten Russlands auf Grund eines Materials von 76648 Geburten, die in den verschiedensten Anstalten unter strenger Antisepsis in den letzten vier Jahren geleitet wurden. Todesfälle durch Vergiftung mit Desinfectionsmitteln wurden nirgends beobachtet. Die puerperalen Erkrankungen betrugen 6,9-10,04 Proc.; die puerperale Sterblichkeit 0,28-0,48 Proc. Die Anwesenheit des lernenden Personales hatte keinen Einfluss auf Morbidität und Mortalität. Bei durchaus gleichmässiger Anwendung der antiseptischen Maassregeln hängt die Grösse der Erkrankungs- und. Sterblichkeitszahlen von der jeweiligen grösseren oder kleineren Zahl pathologischer oder complicirter Entbindungen ab. Grosse Gebäranstalten mit streng durchgeführter Antisepsis sind von grösserem Nutzen als kleine Gebärasyle.

3) Correferent Stadfeld (Kopenhagen) stellt die folgenden Thesen auf: Eine streng durchgeführte Antisepsis berechtigt die 
Gebäranstalten, nicht nur für den Unterricht, sondern auch als wahre Humanitätsanstalten $\mathrm{zu}$ bestehen.

Eine Abzweigung der Gebärenden von der Klinik ist daher heutzutage unnütz, vielleicht sogar gefährlich, weil in den Filialen die Antisepsis schwerer zu überwachen ist.

Auch für die Neugeborenen hat die Einführung der Antisepsis eine Verminderung der septischen Todesarten und des Trismus neonatorum zur Folge gehabt.

Auf die Sterblichkeit in den von Hebammen geleiteten Geburten haben strenge Desinfectionsvorschriften für die Hebammen bereits einen günstigen Einfluss geübt; das Frgebniss muss jedoch noch ein besseres werden. Neben der Asepsis bezw. Antisepsis des helfenden Personales ist die Asepsis der Gebärenden und ihrer Umgebung durchaus erforderlich. Eine sorgfältige Reinigung der äusseren Genitalien ist nothwendig. Prophylaktische Scheidenausspülungen aber sind überflüssig, ja seitens der Hebammen gefährlich. - Für die Desinfection der Kreissenden wird in der Kopenhagener Anstalt ausschliesslich Carbolsäure verwendet. Die äussere Untersuchung muss möglichst genau vorgenommen werden, damit die innere nicht zu oft nothwendig werde. Die Nachgeburtsperiode soll sich nicht ganz selbst ïberlassen bleiben. Mässiges Reiben der Gebärmutter und vorsichtiges Ausdrücken der Placenta soll erlaubt sein. - Fälle von Wochenbettsfieber müssen sofort der Sanitätsbehörde gemeldet werden, damit die betreffende Hebamme desinficirt, bezw. eine Zeit lang ausser Thätigkeit gesetzt werde.

4) Correferent Fritsch (Breslau) plaidirt für folgende Thesen: Bei gesunden Wöchnerinnen unterbleibt jede örtliche Behandlung. Bei niedrigem Fieber (Resorptionsfieber) wird genau beobachtet; zu dieser Beobachtung gehört nur das Thermometer.

Die Indication zu Ausspülungen im Wochenbette hat nur der Arzt zu stellen.

Bei hohem Fieber wird die Gebärmutter ausgespült. Daneben ist der Organismus zu kräftigen durch Stärkung des Herzens und Ankämpfung gegen das Fieber. -

Unter den letzten 1620 Geburten seiner Anstalt hatte Fritsch keinen Todesfall zu verzeichnen. Die Verschiedenheit der antiseptischen Verfahren war ziemlich einflusslos auf die Erkrankungen. 
Besprechung.

Pippingskiöld (Helsingfors) giebt eine Schilderung der Asepsis der Geburten, wie sie sich in der Praxis seit 20 Jahren in Finnland gestaltet hat. Die Hebammen werden streng zur Asepsis angehalten; auf diese Weise werden die Antiseptica so viel wie möglich entbehrlich gemacht. Werden die Lochien übelriechend, so darf die Hebamme Scheidenausspülungen machen. Die Schülerinnen werden sechs Monate lang zur Asepsis erzogen, erst dann werden sie zu den Kreissenden zugelassen. Nach operativen Entbindungen wurden nur bei Blutungen Ausspiulungen der Gebärmutter gemacht; im Wochenbette wurden diese nur bei Zersetzungen in der Gebärmutterhöhle ausgeführt. In den Jahren 1879-1883 sind an puerperalen Erkrankungen gestorben 0,7 Proc., von 1884 bis 1887 nur 0,29 Proc.

Döderlei n (Leipzig) hat bei der Untersuchung der Scheidenabsonderung Schwangerer zwei Typen von Absonderung gefunden. Die gesunde Absonderung enthält Plattenepithelien mit vereinzelten Schleimkörperchen und ausschliesslich Bacillen. Die zweite Form enthält Eiterzellen und Coccen. Die erste Absonderung reagirt stark sauer, die zweite alkalisch. Bei der ersten Gruppe sind Scheidenausspülungen voraussichtlich ibberflüssig, bei der zweiten wahrscheinlich nothwendig. Dies zu entscheiden, muss weiteren Untersuchungen vorbehalten bleiben.

Priestley (London) rühmt ebenfalls die Erfolge, welche die Einführung der Antisepsis in die Gebäranstalten gehabt hat. Gegenüber Galabin bemerkt er, dass er von Sublimatausspülungen 1:2000 üble Folgen gesehen hat; man dürfe dieselben nicht stärker als $1: 4000$ anwenden.

\section{Sitzung am 5. August Vormittags.}

Vorsitzender: Halbertsma (Utrecht).

Leopold (Dresden): 1) Demonstration zur Lehre von den Extrauterinschwangerschaften.

Implantation eines $9 \mathrm{~cm}$ langen Kaninchenfötus in die Bauchhöhle eines anderen Kaninchens. Letzteres nach 60 Tagen getödtet. Bildung eines Fruchtsackes um den skelettirten Fötus.

Fälle von interstitieller Schwangerschaft.

Zwei Fälle von Eileitersschwangerschaft. Bei dem einen be- 
128 Verhandlungen der gynäkologischen Section d. X. internationalen

ginnende natürliche Berstung, bei dem anderen Tubenabort mit Haematokele retrouterina. Laparatomie. Heilung.

Zwei Fälle von Graviditas tubo-ovarica sinistra mit ausgetragener Frucht. Operation zwei Monate nach dem Tode der Frucht. Entfernung der ganzen Fruchtsäcke. Genesung.

Ein Fall von Sehwangerschaft im rudimentären Horne, X. Monat. Laparatomie zwei Monate nach dem Tode der Frucht. Genesung.

Graviditas ovarica dextra, gefunden bei einer 75 jährigen Frau, welche das Steinkind 35 Jahre getragen hatte.

2) Drei wegen Prolapsus totalis gänzlich entfernte Gebärmütter mit Abtrennen grosser Scheidenlappen.

3) Vier nach Freund durch Laparatomie vollständig entfernte carcinomatöse Gebärmütter.

4) Die schwangere Gebärmutter einer Kuh mit injicirten Lymphgefässen.

Barbour (Edinburgh): Demonstration von Gefrierschnitten vom VIII. Monat der Sehwangerschaft und von Kreissenden aus allen drei Geburtsperioden.

Dührssen (Berlin): Demonstration eines Falles von tiefer Cervix- und eines solchen von Scheidendammincision.

Der erste Fall ${ }^{1}$ ) betrifft eine 23jährige I para, zu welcher D. wegen Eklampsie gerufen wurde. Es waren kurz hintereinander neun Anfälle aufgetreten, der Muttermund war für einen Finger durchgängig, die supravaginale Partie eröffnet, der Kopf stand im Beckeneingange. Nach sechs tiefen, $d . h$. bis zum Ansatze an die Scheide geführten Cervixincisionen gelang es leicht, den Kopf mit einer Traction bis auf den Beckenboden zu ziehen und ihn nach einer kleinen Incision in den Eingang über den Damm zu heben. Nach der Geburt traten noch zwei leichte Anfälle auf, weiterhin normales Wochenbett. Das Kind ist prächtig an der Mutterbrust gediehen. Die ersten zwei seitlichen Einschnitte sind unvereinigt geblieben.

Der Fall bewreist, dass man in allen Fällen bei I paren, wo Gefahr für Mutter und Kind es erheischt, also speciell bei Eklampsie, bei alten I paren, bei vorzeitigem Blasensprunge, sobald nur öflentlicht.

1) Der Fall ist hereits in den Therapentischen Monatsheften 1890 ver- 
der supravaginale Theil der Cervix verstrichen ist, die Entbindung in durchaus schonender und ungefährlicher Weise für Mutter und Kind bei noch völlig geschlossenem äusseren Muttermunde vornehmen kann.

In dem zweiten Falle handelt es sich um eine 46 jährige Ipara mit ihrem an der Mutterbrust prächtig gedeihenden Kinde. Hier wurde ich zwölf Stunden nach dem Blasensprunge gerufen. Die Gebärmutter lag der Frucht innig an, es handelte sich um Armvorfall bei abgewichener Schädellage. Oberhalb des Scheidenansatzes befand sich eine Strictur, welche die Hand zunächst nicht durchtreten liess. Es wurden nun drei Einschnitte in den Muttermundssaum gemacht - um jeden Widerstand seitens desselben aufzuheben - und ebenfalls ein rechtsseitiger Scheidendammeinschnitt angelegt, da der Vortragende durch das erste Eingehen mit der ganzen Hand die Columna rugarum posterior an der hinteren Commissur abgerissen hatte und weitere unvermeidliche Zerreissungen vermeiden, sowie die Ausziehung möglichst erleichtern wollte. Der Durchtritt durch die Scheide war jetzt ungemein leicht, und nach Reposition des Armes gelang es auch mit einiger Mühe, durch die Strictur zu kommen und die Wendung zu machen. Die sofort angeschlossene Ausziehung gelang leicht bis auf die Entwickelung des grossen und festen Schädels. Das tief scheintodte, $3750 \mathrm{~g}$ schwere Kind wurde nach einer Stunde völlig wiederbelebt. Der Scheidendammeinschnitt wurde in seiner oberen Hälfte durch fortlaufende Catgutnaht, in der unteren durch Silkwormnähte geschlossen, nachdem zum Zwecke der völligen Blutstillung der Utero-vaginalcanal mit einem sterilisirten, in 3 proc. Carbolsäurelösung ausgedrückten Gazestreifen tamponirt war. Die Wunde heilte durch erste Vereinigung, das Wochenbett verlief normal. Der Vortragende ist der Ueberzeugung, dass ohne den Scheidendammeinschnitt in diesem Falle die Entbindung von einem lebenden Kinde unmöglich gewesen wäre.

Die beiden Fälle stellen Methoden dar, welche den Widerstand seitens der Weichtheile völlig aufheben und die schonende und ungefährliche Entbindung von lebenden Kindern in Fällen gestatten, wo man bis jetzt die Kinder einfach absterben liess, oder perforirte, oder gewaltsame Ausziehungen mittels der Zange machte, oder, wie $\mathrm{Halbertsma,} \mathrm{den} \mathrm{Kaiserschnitt} \mathrm{ausführte.}$ Letzteren möchte der Vortragende neben seiner Methode nur für Archiv f. Gynäkologie. Bd. XXXIX. Hft. 1. 


\section{Verhandlungen der gynäkologischen Section d, X. internationalen}

die Fälle gelten lassen, wo der supravaginale Theil der Cervix noch geschlossen ist.

Neugebauer, der Jüngere (Warschau): Zur Lehre von der Pathologie der Wirbelsäule und des Beckens.

Der von Neugebauer geschilderte Fall betrifft eine Selbstmörderin, welche infolge eines Sprunges aus dem Fenster einen frischen Bruch des letzten Lendenwirbels, und zwar der Portio interarticularis seines Bogens, erlitten hatte. Noch unlängst hatte Lambl einen solchen Bruch als ein Erzeugniss erfinderischer Phantasie hingestellt. Ausserdem hatte die schwangere Person eine Zerreissung der Gebärmutter erlitten. Das Kind lag frei in der Bauchböhle. Die Eihäute am unteren Eipole waren unverletzt.

Olshausen (Berlin): Demonstration von Kranken.

Olshausen zeigt einen Fall, bei welchem er vor zwei Jahren den schwangeren retroflectirten Uterus wegen dex Unmöglichkeit der Reposition (osteomalakisches Becken) entfernt hat. Die Einleitung des künstlichen Abortus war in diesem Falle unmöglich, weil der Muttermund zu hoch stand.

In dem zweiten Falle war zwei Mal im Laufe eines Jahres wegen Extrauterinschwangerschaft laparatomirt worden. Das erste Mal wurde bei rechtsseitiger geborstener Eileitersschwangersehaft das fast reife Kind lebend entwickelt. - Kaum ein Jahr später handelte es sich um Berstung des linken Eileiters. Die Frau kam sofort in die Klinik und wurde sogleich operirt. Man fand $2500 \mathrm{~g}$ Blut in der Bauchhöhle. Dieses wurde entleert, und die Frau genas auch diesmal schnell.

Im dritten Falle wurde der prolabirte Uterus mit einem grossen Theile der Scheide nach der Methode von Fritsch entfernt. Olshausen hat diese Operation drei Mal gemacht.

Calderini (Parma): Demonstration eines Myoma colli uteri von der Grösse zweier Fäuste, herstammend von einer Kreissenden, bei welcher die Wendung und Ausziehung gemacht wurde. Hierbei wurde die Geschwulst herabgezogen und der Stiel abgebunden.

v. Ott (Petersburg): Gesetz der Periodicität der phy siologischen Functionen im weiblichen Organismus.

v. Ott constatirt, dass die Energie der Functionen im weiblichen Organismus sich vor Beginn der Menstruation steigert, dann aber bei oder unmittelbar vor Beginn der Blutung abnimmt. 
Genaue Untersuchungen über Wärmeausstrahlung, Muskelkraft, Lungencapacität (Inspirations- und Exspirationskraft), sowie über das Verhalten des Nervensystems auf Grund der Reactionszeit des Sehnenreflexes bei gesunden und unter gleichen Verhältnissen stehenden Frauen haben ergeben, dass die vermuthete Wellenbewegung in den Functionen des weiblichen Organismus ein nunmehr wissenschaftlich begründetes und physiologisches Gesetz ist, und dass diese von der Menstruation abhängt.

Marocco (Rom) zeigt einen neuen Gazeträger vor für die Tamponade der nicht puerperalen Gebärmutter, der den Cervicalcanal zu gleicher Zeit erweitert.

Fritsch (Breslau): Vorlegung von fünf Präparaten, welche durch Exstirpation der Gebärmutter und der Scheide bei Prolapsus gewonnen sind. Von der Scheide sind gewaltig grosse Stücke entfernt worden.

\section{Sitzung am 5. August Mittags.}

Vorsitzender: Péan (Paris).

II. Allgemeines Thema: Exstirpatio uteri vaginalis.

Referent Williams (London) betont, dass Krebs der Gebärmutter an und für sich eine Indication zur Totalexstirpation der Gebärmutter ist. Aber nicht alle Fälle sind für diese Operation geeignet. Die Totalexstirpation soll man nach Williams nur vornehmen, wenn wirklich radicale Heilung zu erhoffen steht; als eine Palliativoperation ist sie zu schwer. In den meisten Fällen ist die hohe Amputation die geeignete Operation. Die Statistiken sind noch zu ungenügend, um den Schlusis zu gestatten, dass die Erfolge nach der Totalexstirpation besser sind, als nach der hohen Amputation.

Correferent Schauta (Prag): Die Hauptindication für die vaginale Exstirpation bildet der Krebs der Gebärmutter. Als untere Grenze der Indication der Totalexstirpation bei Carcinom ist jeder Fall von diagnosticirbarem Uteruscarcinom anzusehen, gleichgültig, ob das Carcinom localisirt ist an Portio, Cervix oder Collum. Als obere Grenze gilt die Möglichkeit, im Gesunden zu. operiren. Schauta verwirft die hohe Amputation. Weitere Indicationen für die Totalexstirpation sind: Myome, Prolapse, stets rückfällige glanduläre Endometritis. Unter 65 Operationen verlor Schauta 5 (7,6 Proc.). 47,3 Proc. blieben zwei Jahre ohne Recidiv (s. den ausführlicheren Bericht in diesem Hefte, S. 115). 
132 Verhandlungen der gynăkologischen Section d. X. internationalen

Correferent Pozzi (Paris) schliesst sich den Ausführungen von Schauta an: Man soll den Uterus total exstirpiren, sobald Krebs diagnosticirt ist. Die Totalexstirpation ist nicht gefährlicher als die Amputation des Collum, zumal die hohe Amputation. Die Totalexstirpation ist vorzuziehen, weil es unmöglich ist, festzustellen, ob die Krankheit das Collum uteri nicht nach oben überschritten hat. Die Totalexstirpation ist auf die Fälle zu beschränken, wo die Krankheit den Uterus nicht überschritten hat. Im anderen Falle ist das palliative Verfahren einzuschlagen. Was die Technik der Operation anbetrifft, so ist die Umstülpung des Uterus zu unterlassen, um eine Infection der Wunde zu vermeiden. Die Partienligatur ist der Forcipressur vorzuziehen. Letztere soll nur ein Nothbehelf bleiben. Die Forcipressur begünstigt Verletzungen der Blase, der Harnleiter, des Mastdarmes und späterhin Verschwärung der Eingeweide und secundäre Hämorrhagie. Sie verengert das Operationsfeld und verhindert die Entfernung der Anhänge, die nothwendig sein kann (Pyosalpinx). Dadurch, dass die Forcipressur die gefassten Gewebe zum Absterben bringt, kommt sie mit einer strengen Antisepsis in Widerstreit.

Correferent 01 shausen (Berlin) führt folgende Thesen aus :

1. Die vaginale Totalexstirpation des Uterus ist bei Carcinom des Organes und in einzelnen Fällen anderer Krankheiten eine oft segensreiche Operation, die nicht wieder aus der Reihe der gynäkologischen Operationen verschwinden darf.

2. Sie ist zur Zeit, wegen der oft spät gestellten Diagnose auf Carcinom, erst in der grossen Minderzahl der Fälle von Carcinoma uteri anwendbar.

3. Die primären Erfolge müssen verschieden sein nach der Ausdehnung, welche man den Gegenanzeigen der Operation giebt. Doch lässt sich selbst bei weit gehender Indicationsstellung die Sterblichkeit auf 10-15 Proc. herabdrücken.

4. Die endgültigen Ergebnisse, d. $h$. die dauernden Heilungen, sind natürlich ungleich ungünstiger; doch wird sich durch zeitigere Diagnose und Operation ein besserer Erfolg als bisher erzielen lassen.

5. Die nicht radical Geheilten haben nach der Operation im allgemeinen weit geringere Beschwerden, als nicht Operirte, was sich aus dem Fehlen der Blutungen und der Absonderungen erklärt. Ausnahmen hiervon kommen vor.

6. Der Rückfall tritt selten in der Scheide auf, gewöhnlich in den Parametrien oder in Organen der Unterleibshöhle. 
7. Was die Ausführung der Operation betrifft, so ist für die grosse Mehrzahl der Fälle die Entfernung durch die Scheide die beste Methode. Sie kann nicht durch die sacrale, parasacrale oder perineale Schnittführung ersetzt werden. Nur in einzelnen Fällen, besonders bei weiter Verbreitung der Neubildung auf die Scheide, wird der perineale Schnitt (nach Zuckerkandl) vielleicht vorzuziehen sein.

8. Für die Technik der vaginalen Exstirpation empfehlen sich folgende Regeln:

Genaue, vorgängige Desinfection des Carcinoms. Möglichst weite Umschneidung des Carcinoms in der Scheide. Schnelle stumpfe Auslösung der Cervix mit möglichster Beschränkung von Gefässligaturen.

Eröffnung des Cavum Douglasii vor Anlegung der ersten Massenligatur. Anlegung der Massenligaturen für das erste Ligamentum latum vom Carum Douglasii her.

Die am weitesten erkrankte Seite ist zuletzt abzubinden. Nach Auslösung der Gebärmutter schliesse man das Bauchfell und die Scheide durch die Naht, womöglich unter ausschliesslicher Anwendung von Catgut und Versenkung der Stümpfe. Alsdann gestaltet sich die Heilung am einfachsten und fast völlig durch erste Vereinigung.

Von 40 von Olshausen in den letzten drei Jahren Operirten waren 19 (47,5 Proc.) über zwei Jahre nach der Operation noch rückfallsfrei, während die Ergebnisse von 1880 - 1887 weniger günstig waren: Von 56 Fällen 6 rückfallsfrei, was zum Theile durch zu weite Indicationsstellung bedingt war.

Besprechung.

Landau (Berlin) empfiehlt für die Technik der Operation die Forcipressur, die für die Fälle von fixirter Gebärmutter allein anwendbar ist. Die Forcipressur ist noch anwendbar in Fällen, wo die Krankheit die Gebärmutter überschritten hat. Landau hat von 35 mit Forcipressur Operirten 3 verloren, davon 1 an Coma diabeticum, 1 an Sepsis, 1 an lleus.

Saiaitzky (Moskau): Zur Frage der Hysterektomia vaginalis wegen Carcinoma (auf Grund von 65 Fällen aus eigener Erfahrung und den übrigen in Russland operirten). Saiaitzky tritt für eine ausgedehnte Anwendung der Totalexstirpation auch bei weit vorgeschrittenen Fällen ein. Als Abänderung der vaginalen Exstirpation empfiehlt er bei grosser Gebär- 
mutter die Spaltung derselben nach P. Müller und das Kraske' sche Verfahren. Er empfiehlt, die Scheide nicht zu schliessen. Von 238 Operationsfällen starben 30 (12,6 Proc.). Rückfälle traten in 43 Fällen auf. Eine Kranke ist seit sieben Jahren rückfallsfrei. $7 \mathrm{Mal}$ entstand eine Vesico-vaginalfistel, $1 \mathrm{Mal}$ eine Ureterenfistel. Mit zunehmender Uebung hat die Zahl der Todesfälle, auf das Hundert berechnet, bedeutend abgenommen. Bei genügender technischer Erfahrung und strenger Wahrung der Antisepsis ist die Operation fast vollständig gefahrlos.

Martin (Berlin): Die Anzeige der Totalexstirpation ist nicht zu beschränken auf Carcinoma oder bösartige Erkrankungen iberhaupt, sondern auszudehnen auf alle Fälle, in denen die Gebärmutter ihre Trägerin unfähig zum Leben oder für den Erwerb macht. Doch soll die Exstirpation nur vorgenommen werden, wenn die Gebärmutter ganz entfernt werden kann. Die Methode der Exstirpation ist gleichgültig. Martin schliesst neuerdings die Wunde in der Scheide vollständig und benutzt zur Unterbindung Catgut. Die endgültigen Ergebnisse lassen sich erst nach weiteren jahrelangen Beobachtungen feststellen.

Kaltenbach (Halle): Das sacrale Verfahren gestattet eine bessere Freilegung des Operationsfeldes, aber keine ausgedehntere Entfernung des Erkrankten. Für wesentlich hält Kaltenbach den Verschluss der Bauchhöhle. Er verlor unter 80 Fällen mit Schluss des Bauchfelles nur 2.

Düvelius (Berlin) hebt hervor, dass er in Berlin als der Erste für die unterschiedslose Totalexstirpation der Gebärmutter bei Carcinoma aufgetreten sei.

Kugelmann (Hannover) erwähnt einen Fall von Carcinoma bei einer Kranken, die jetzt, fünf Jahre nach erkannter Unmöglichkeit der Totalexstirpation, noch am Leben ist. In Bezug auf Verhütung des Carcinoma scheint ihm eine frühzeitige Behandlung der Geschwüre des Scheidentheiles nothwendig.

Czeruy (Heidelberg) betont den Wandel in den Ansichten über den Werth der Totalexstirpation. Dieselbe wird jetzt auch im Auslande anerkannt. Dies ist eine Wirkung der internationalen Congresse. - Das sacrale Verfahren ist für die Fälle von Erkrankung der Parametrien und der Scheide vorzuziehen.

Fränkel (Breslau) berichtet über einen Fall von Amputatio portionis vaginalis wegen Carcinoma bei einer 34jährigen Frau. 
Nach acht Jahren Rückfall und Tod ron einer linsengrossen Geschwürsfläche aus, die sich nie geschlossen hatte. Der Fall spricht für die Totalexstirpation auch in Fällen von gut begrenztem, intravaginal absetzbaren Carcinoma portionis vaginalis.

Péan (Paris) kommt zu folgenden Schlüssen: Wenn es sich um eine epitheliale Geschwulst des Collum uteri handelt, so kann man das erkrankte Collum in Stz̈cken entfernen, bevor man es herunterzieht, und in der Weise ausschneiden, dass das Bauchfell nicht durch die Trümmer der Geschwulst verunreinigt werden kann. Betrifft das Carcinoma das Corpus uteri, so muss man zunächst das Collum wegschneiden und dann das Corpus mit dem pathologischen Erzeugnisse durch Morcellement entfernen. - In dem Falle, wo die Gebärmutter erweicht oder theilweise durch Eiterung zerstört ist, ist ihre Entfernung leicht, selbst wenn sie von Adhärenzen umgeben ist. Dasselbe ist der Fall, wenn die Gebärmutter der Sitz eines bis mannskopfgrossen Myoma ist. Péan nimmt die Entfernung der Gebärmutter mit Hülfe seiner Pinces vor; also ohne Ligatur, ohne nachfolgende Hämorrhagie, ohne Verletzung der Nachbarorgane - falls der Operateur geübt ist. Nach diesem Verfahren hat Péan bei 60 Fällen von vaginaler Hysterektomie wegen Vereiterung oder Verwachsung keinen und unter 200 derartigen Operationsfällen wegen Myoma nur vier Todesfälle gehabt. Die Kranken standen am 10. Tage auf und waren am 15. Tage geheilt. - Im Schlussworte betont Péan die Bedeutung der neueren Gynäkologie für die Verrollkommnung der Chirurgie überhaupt.

\section{Sitzung am 5. August Nachmittags.}

Vorsitzender: Simpson (Edinburgh).

Schatz (Rostock): Die Ursache der Drehung des Fruchtkopfes um die Achse des Beckencanales. Der grosse eiförmige Kopf und der an dessen Aequator ansitzende Hals der menschlichen Frucht bewirken, dass Kopf und Rumpf nicht regelrecht hintereinander durch das Becken treten können. Wenn der Kopf noch nicht ganz in das Becken eingetreten ist, so stemmt sich auch schon der mit dem Kopfe durch den kurzen Hals verbundene Brustkorb gegen den Beckeneingang an. Da nun der Brustkorb nicht beliebig mit eintreten kann, so hält er mittels des Halses auch den Kopf - diesen aber nur an der 
136 Verhandlungen der gynäkologischen Section d. X. internationalen

Nackenseite - zurück. Dieser Rückzug ist es, welcher eine Drehung des Kopfes bewirkt, sobald sich bei seinem synklitischen Fortschreiten sein Aequator gegen den Beckeneingang schief stellt. Dabei kommt die Ansatzstelle des Halses an den Kopf stets in die Concavität des Beckencanales. Je schwieriger oder je weniger möglich der gleichzeitige Eintritt von Kopf und Brustkorb in das Becken ist, um so sicherer entsteht die Drehung - bei grossem Kinde oder bei Gesichtslage. Diese Theorie hat Schatz durch tokomechanische Apparate bewiesen.

Zweifel (Leipzig): Beiträge zur Lehre rom Geburtsmechanismus unter Demonstration von Gefrierdurchschnitten durch den Körper einer Hochschwangeren. $Z_{w e i f e l}$ giebt zunächst eine Beschreibung des Schnittes und empfiehlt die vom unteren Rande der Schamfuge parallel zum Beckeneingange verlaufende Ebene - die nach Hodg e sogenannte Parallelebene - Beckenweite zu nennen. Die erste Drehung die Senkung der kleinen Fontanelle - ist die Folge von Druck und Widerstand. Bei auffallend weitem Becken bleibt die Senkung aus. Die zweite Drehung beginnt, sobald der grösste Umfang des Kopfes in die parallele Beckenweite eingetreten ist, wofür der Gefrierschnitt von Braune spricht. Sie hängt also zunächst von der Configuration des Beckens ab.

Die Ansicht von Olshausen, dass die Drehung des Rückens das Primum movens sei, wird durch Schnitte widerlegt, bei denen die Drehung der Pfeilnaht schon begonnen hatte, während der Rücken noch vollkommen quer stand. Weiterhin sind für die zweite Drehung von Wichtigkeit - wie Veit betont hat - die Weichtheile der Beckenenge. Die klinische Prüfung dieser Theorie ist nur durch eine genaue Feststellung des jedesmaligen Kopfstandes möglich. Dann lässt sich feststellen, dass vor der zweiten Drehung der grösste Umfang des Kopfes in der zweiten Hauptebene liegt. Erst nach völliger Eröffnung des Muttermundes ist die zweite Drehung vollendet, bei welcher die kleine Fontanelle vom unteren Rande des Ramus superior ossium pubis bis zum Ramus inferior ossium pubis herabrückt. Die sogenannte vierte Drehung - diejenige um die fronto-occipitale Achse - hängt mit der stärkeren Zusammenziehung an der von der Placenta nicht besetzten Wand zusammen. Sitzt die Placenta vorn, so wird der kindliche Rumpf nach hinten abgebogen; die hintere Schulter berührt den Kopf, und das vordere Scheitelbein rollt an der Scham- 
fuge tiefer. Diese Abbiegung der Kindslänge erklärt auch ungezwungen die Entstehung der Vorderscheitel- und Gesichtslagen.

Besprechung.

Barbour (Edinburgh) betont, dass in seinen Gefrierdurchschnitten der Kopf nach vorn gedreht ist, der Rücken aber nicht, was gegen die Theorie von Olshausen spricht.

J. Veit (Berlin) vertheidigt den von ihm gewählten Namen „Hauptebene“ für die zweite parallele Ebene nach Hodge, weil in dieser Ebene der Geburtsmechanismus beginnt. Nur das enge Becken hat einen Eingangsmechanismus. Die Senkung der kleinen Fontanelle wird durch die der Frucht innewohnende Wachsthumsrichtung bedingt. Die widersprechende Beobachtung Zweifel's ist bei engem Becken gemacht.

Dem gegenüber hebt $\mathrm{Zweifel}$ hervor, dass in seinem Falle das Becken ein normales sei. Bei Schwangeren stehe der Kopf mit seinem grössten Umfange noch über dem Beckeneingange.

Pestalozzi (Pavia): Graphische. Darstellung des fötalen Herzimpulses. Es handelte sich um eine Zwillingsschwangerschaft, wo der Herzstoss der zweiten Frucht, welche quer lag, in der Zahl von 140 in der Minute gefühlt werden konnte. Mittels eines Sphygmographen konnte der Herzstoss der Frucht aufgenommen werden. Während der Wehe wurde der Fötalpuls nicht verändert.

Skutsch (Jena): Zur Beckenmessung. Skutsch zeigt das neueste Modell seines Beckenmessers zur into-externen Messung vor, welcher die unmittelbare Messung der inneren Beckendurchmesser gestattet.

Balandin (Petersburg) hat schon früher darauf aufmerksam gemacht, dass die normale Conjugata keine Bedeutung für den Geburtsmechanismus hat. Nur bei tiefem Stande kann der Vorberg auch bei normaler Weite des Beckens den Kopf quer ablenken. Bei dem von $\mathrm{Z}$ weifel vorgelegten Becken steht der Vorberg sehr tief. Das Becken ist daher nicht regelmässig. Ein tief liegender Vorberg wird bei gleicher Grösse der Conjugata dem Kopfe mehr Widerstand bieten, als ein hoch liegender.

Budin (Paris) beschreibt einen Fall von rhachitischem Becken, wo er durch genaue Messung auch der queren Durchmesser des Beckens den richtigen Zeitpunkt für die Einleitung der künstlichen Frïhgeburt im Beginne des achten Monates traf und das Kind von selbst geboren wurde. 
138 Verhandlungen der gynäkologischen Section d. X. internationalen

Küstner (Dorpat): Ueber die Entstehung amniotischer Bänder. Küstner berichtet über einen Fall, wo bei dem ausgetragenen lebensfrischen Kinde einer gesunden Zweitgebärenden das Amnios in früher Fötalperiode geplatzt, vom Chorion bis zur placentaren Einsenkung der Nabelschnur herangezogen und zu einem nach dem Ende zu sich immer mehr verjüngenden Strange aufgedreht worden war. Dieser Strang hatte sich um die Gliedmaassen des Kindes gewickelt und viele Amputationen an diesen veranlasst. Der spiralig um die Einsenkung der Nabelschnur gewundene Amniosstrang hatte ausserdem Hautödeme verursacht. An der rechten Hand zeigten die Amputationsstümpfe noch Granulationen. Das Bersten des Amnios fand vermuthlich am Ende des zweiten Monates statt, wo Erscheinungen eines beginnenden Abortus auftraten.

Stuart Nairne (Glasgow): Untersuchungen über einige Ursachen von Incontinentia urinae bei Frauen und ihre chirurgische Behandlung. Stuart Nairne schildert zwei Fälle, in denen die Harnröhre durch eine gefässreiche sammtartige Membran verengert war. Die Behandlung bestand in Durchschneidung der Membran und Cauterisation. In beiden Fällen trat Heilung ein.

Neugebauer d. Ae. (Warschau) berichtet über eine Heilung von Inversio uteri durch hydrostatischen Druck.

\section{Sitzung am 6. August Vormittags.}

Vorsitzender: A. Martin (Berlin), da Parvin (Philadelphia) nicht erschienen.

Schultze (Jena): Bildliche Darstellung des gynäkologischen Tastbefundes. Dieselbe fördert den Anfänger im Untersuchungscurse, den Zuhörer der Klinik. Sie erleichtert den Bericht an Collegen. Sie giebt den Befund genau wieder und erfordert deswegen auch eine genauere Untersuchung. Sie erleichtert den Ueberblick und die Vergleichung, wenn die Kranke wiederholt um Rath fragt. Die Schemata sind, wie Schultze des Näheren nachweist, vollständig der Wirklichkeit entsprechend. Von Hertel in Breslau werden sie in Stempelform geliefert.

Outter (New York) legt ein Pessar gegen Prolapsus vor.

Mo ore Madden (Dublin) erläutert einen Universalleuchter für Specula und ein neues Pessar. 
H. W. Freund (Strassburg): Erklärung von Schnitten durch das normale und durch das erkrankte Beckenbindegewebe.

Edebohls (New York): Vorführung eines aseptischen Tisches für Laparatomien, eines selbst haltenden rinnenförmigen Speculum vaginae und eines verbesserten Schlusses für chirurgische Instrumente.

Kaltenbach (Halle): Erfahrungen über Sarkomata uteri. Kaltenbach zeigt sieben Gebärmütter vor, die er in den letzten drei Jahren wegen Sarkoma vollständig entfernt hat, und beschreibt ihre anatomischen Eigenthümlichkeiten. $4 \mathrm{Mal}$ fand sich ein Sarkoma der Cervicalschleimhaut; $6 \mathrm{Mal}$ wurde die Gebärmutter von der Scheide aus, $1 \mathrm{Mal}$ nach Freund entfernt. Von den 7 Kranken sind 2 innerhalb 4-7 Monaten gestorben. Die übrigen sind rückfallsfrei; einige davon schon über $21 / 2$ Jahre. In einem achten Falle machte Kaltenbach die supravaginale Amputatio von der Bauchhöhle aus bei einem 15jährigen Mädchen, das $1 \frac{1}{2}$ Jahre später an einem Rückfalle zu Grunde ging. In einem neunten nicht $\mathrm{zu}$ operirenden Falle mit peritonealen Metastasen war die Frau ein Jahr vorher von einer sehr grossen Blasenmole entbunden worden. Da ein gleicher Fall von Leopold vorliegt, scheint ein ätiologischer Zusammenhang der beiden Krankheiten zu bestehen. Dies wäre ein neuer Beweis für den wirklich bösartigen Charakter des Myxoma chorii.

Routh (London): Vorlegung eines intrauterinen Pessars, eines abgeänderten Ecraseurs und eines Stethoskopes für vaginale Auscultation bei Schwangeren.

Sabatier: Erläuterung eines Beckens mit doppelter Synostose der Articulatio sacro-iliaca ohne quere Verengerung. Als Ursache der Synostose sieht Sabatier Rhachitis an.

Bröse (Berlin): Erläuterung von medicinisch-elektrischen Apparaten, welche mit der von Dynamomaschinen erzeugten Elektricität gespeist werden.

Hillischer (Wien) und von Swiecicky (Posen): Vorlegung von tragbaren Apparaten zur Anästhesie mit

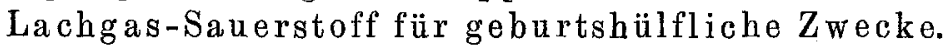


140 Verhandlungen der gynälkologischen Section d. X. internationalen

\section{Sitzung am 6. August Nachmittags.}

Vorsitzender: Slavjansky (Petersburg).

Moore Madden: Die Behandlung der obstructiven Dysmenorrhoe. Moore Madden beschreibt einen Dilatator, welcher die Erweiterung von oben her beginnt. Zur operativen Behandlung der Cervicalstenose gebraucht Moore Madden das Simpson'sche Metrotom.

Derselbe: Ueber Cystitis. Empfehlung der Dilatatio urethrae, Curettement der Blasenschleimhaut bezw. Anwendung von Carbolglycerin auf die Blasenschleimhaut.

Th. Landau (Berlin): Einige Bemerkungen über das Thure-Brandt'sche Verfahren. Landau stellt das Brandt'sche Untersuchungsverfahren über die bisher bekannten. Als eine erwähnenswerthe Thatsache hebt er hervor, dass bei der palpatorischen Massage die myomatöse Gebärmutter sich ungleichmässig, die Gebärmutter mit chronischer Metritis sich gar nicht zusammenzieht. Ferner lässt sich oft eine vorübergehende Anfüllung der Eileiter mit Flüssigkeit feststellen.

Döderlein (Leipzig) lobt die guten Erfolge der Massage bei Retroflexio uteri, indem die regelmässige Lage durch dieselbe dauernd erzielt wird, und zwar durch die einfache Massage der Gebärmutter allein. Nachdem die Gebärmutter in Anteflexio gebracht worden war, hielt er sie längere Zeit in dieser Stellung fest und massirte sie, bis sie ganz hart wurde.

Skutsch (Jena) betont, dass das Untersuchungsverfahren von Brandt nur wenig von dem Schultze'schen abweicht, und dass die gynäkologische Massage nur der Meister in der gynäkologischen Untersuchung üben dürfe.

Kugelmann (Hannover) hat frische und alte parametritische und perimetritische Exsudate durch in den Mastdarm eingelegte Opiumzäpfchen und durch Jodkalium verschwinden sehen und hält daher die Massage für überflüssig, ja für schädlich, weil sie eine Art Onanie ist.

Th. Landau (Berlin) hält Skutsch gegenüber seine Behauptung aufrecht, dass die palpatorische Massage einen wesentlichen Fortschritt in der Diagnostik darstellt.

Doléris (Paris): Die physiologische Grundlage in der Gynäkologie - Nothwendigkeit einer conservativen Chirurgie. In der Gynäkologie muss die Function der 
Organe erhalten bleiben; daher soll man z. B. bei Endometritis nicht ätzen, wodurch die Uterusschleimhaut zerstört werden kann, sondern curettiren. Noch weniger soll man bei Erosionen oder Ektropium ätzen, sondern das erkrankte Gewebe mit dem Messer entfernen. Bei der Verlängerung des Collum mit verstärkter Anteflexion muss die physiologische Form, und zwar durch das Messer, wiederhergestellt werden. Die Herstellung der physiologischen Form wird bei Lageveränderung mit Prolapsus erreicht durch plastische Verengerung der Scheide, verbunden mit Verkürzung der verlängerten Ligamenta rotunda. $7 \mathrm{Mal}$ sah Doléris bei 60 so Operirten Schwangerschaft eintreten in dem kurzen Zeitraume von drei Jahren. Von 145 Fällen von Salpingitis und Oophoritis, sowie peritonitischen Exsudaten wurde in 56 Fällen durch Allgemeinbehandlung eine solche Besserung erzielt, dass eine operative Behandlung verweigert wurde. Unter 30 Fällen wurde durch Operation am Collum und durch Behandlung der Innenfläche der Gebärmutter bei 22 Fällen ein befriedigender Erfolg erzielt; bei den übrigen 8 trat durch die Operation am Collum keine Besserung ein; es wurde daher laparatomirt. $25 \mathrm{Mal}$ wurde die Erkrankung der Anhänge durch Herstellung der richtigen Lage beseitigt, und in 39 weiteren Fällen wurde die Laparatomie gemacht. Doléris ist also der Ansicht, dass sich Laparatomien durch kleinere Eingriffe, wie das Curettement, Erweiterung u. s. w. oft ersetzen lassen.

Rein (Kiew): Asepsis oder Antisepsis bei Laparatomien? Rein kommt auf Grund sorgfältiger klinischer und bakteriologischer Untersuchungen bei 87 Laparatomien mit 2,2 Proc. Sterblichkeit zu dem Ergebnisse, dass die Sterilität der Wunde erreichbar ist, und zwar durch eine Verbindung des aseptischen mit dem antiseptischen Verfahren. Vorläufig lässt sich die Antisepsis noch nicht entbehren. Nur bei Erkrankungen parenchymatöser Organe - namentlich der Nieren - und bei Probeschnitten ist vielleicht jetzt schon ,die aseptische Form des Sterilisationsverfahrens der Wunden" angezeigt.

Sänger (Leipzig): Ueber die Drainage der Bauchhöhle bei Laparatomien. Was die Form der Drainage betrifft, so bevorzugt Sänger die combinirte Gazeglasröhrendrainage mittels krummer und mit zahlreichen Oeffnungen versehener Glasröhren. Natürlich müssen die einzuführenden Gazestreifen sterilisirt sein. Die in dieser Weise ausgeführte Drainage wirkt ausgezeichnet. Die Drainage ist angezeigt bei chronischer Entzündung 
oder bedeutender Verletzung des Bauchfelles, bei Gefahr von Blutung aus flächenhaften Verwachsungen, nach Berstung von mit Eiter gefüllten Säcken. Die Drainage wirkt ferner günstig durch die Abkapselung, welche sie erzielt. Die Drainage der Bauchhöhle stellt ein Sicherheitsventil dar. Sie macht die vaginale Drainage überflüssig. Nach der Bekanntmachung von Lande (siehe dieses Archiv, Bd. XXXVI, S. 460) hat Sänger noch 14 Mal drainirt mit nur einem Todesfalle an Erschöpfung.

Cushing (Boston): Drainage nach Laparatomie. Cushing glaubt, dass die Drainage hauptsächlich von denen angewendet wird, welche einen kurzen Einschnitt machen und die Bauchhöhle mit heissem Wasser ausspülen. Die Hauptanzeige besteht in der Vermeidung von Flüssigkeitsansammlung im Becken. Dies wird durch das Drainagerohr erreicht. Allerdings wird durch die Drainage die Nachbehandlung verwickelt. Die Erfahrung lehrt, dass die, welche starben, hätten drainirt werden müssen, und dass die Drainirten weniger oft sterben als die Anderen. Ausser bei Verwachsungen oder Zerreissungen des Bauchfelles oder bei Vorhandensein von Eiter, Blut, Urin soll man drainiren bei Verletzungen der Eingeweide und der Blase, sowie bei Verschwärungen dieser Organe. Ferner ist die Drainage angezeigt bei Exsudaten, bei Höhlenbildung mit festen Wandungen, bei Shoc, welcher schnelle Vornahme der Operation erheischt. Gegenanzeigen giebt es nicht.

Lawson Tait (Birmingham) betont, dass nur todte oder absterbende Stoffe der Zersetzung anheimfallen. Daher müssen sowohl diese wie auch ergossenes Blut oder Serum entfernt werden. Dieses Verfahren ist viel sicherer als die Zerstörung der Keime. Ferner zeigt das Drainagerohr sofort eine secundäre Blutung an. Ausserdem ist das Drainagerohr nothwendig, wo der Mastdarm oder andere Eingeweide zum Zwecke der Vollendung der Operation verletzt werden müssen.

Bantock (London) giebt nicht zu, dass das Drainagerohr immer Verwachsungen mache. Bei früher mit Drainage behandelten Fällen von Laparatomie hat Bantock bei Wiederholung der Operation keine Spur von Verwachsungen gefunden. In der Flüssigkeit des Drainagerohres hat Bantock niemals Keime gefunden. Das Wesen der Drainage liegt darin, dass man den überall vorhandenen Mikroorganismen den Nährboden entzieht. Zu Bauchbrïchen giebt die Drainage nicht Anlass. 
Lloyd Roberts (Manchester): Zwei Fälle von Laparatomie wegen tuberkulösen A scites. (Empfehlung der Drainage für diese Fälle.)

Boldt (New York): Peritonitis, hervorgerufen durch Berstung eines Ovarialhämatoms. Laparatomie Heilung. Boldt führt die Entstehung des Hämatoms auf ein Endotheliom mit Uebergang in Angiom zurück. Derartige Fälle sind von Jones beschrieben worden. Das Epitheliom entwickelt sich in der Wand geborstener Graaf'scher Follikel.

\section{Sitzung am \%. August Vormittags.}

Vorsitzender: Pasquale (Rom).

von 0tt (Petersburg): Erklärung einer elektrischen Beleuchtungslampe, für welche die Elektricität durch Accumulatoren geliefert wird.

A uvard (Paris): Behandlung von Schrunden. Die Vorbeugungsmassregeln gegen die Entstehung von Schrunden bestehen in der Erzeugung einer hervorragenden Warze und in der Kräftigung derselben. Erstere wird durch einen von A u vard angegebenen Apparat (Téterelle biaspiratrice) erzielt, letztere durch Waschungen mit Alcohol im letzten Monate der Schwangerschaft und im Beginne des Stillens. Die Behandlung der Schrunden selbst besteht in der Anwendung von antiseptischen und Vernarbung befördernden Mitteln, sowie in der Anwendung des oben genannten Apparates.

Auvard: Vorlegung eines neuen Embryotoms (Embryotome céphalique combiné). Dasselbe besteht aus einem gewöhnlichen Kranioklaster, dessen inneres Blatt zur Perforation benutzt werden kann. Macht die Ausziehung mit diesem gewöhnlichen Kranioklaster Schwierigkeiten, so führt man auf der freien Seite des Kopfes ein drittes Blatt ein, welches durch Anwendung der Flügelschraube den Kopf zermalmt. Das ganze Instrument ist sehr einfach und schützt völlig vor dem Abgleiten bei der Zermalmung.

W. S tew art (Philadelphia): Vorlegung eines verbesserten Handgriffes an der Zange. Das Princip seiner Zangenconstruction besteht darin, dass die Griffe sich nicht kreuzen, sondern parallel nebeneinander verlaufen. 


\section{Verhandlungen der gynähologischen Section d. X. internationalen}

Dührssen (Berlin): Ueber Operationen an der Portio vaginalis. Dührssen berichtet über eine Reihe von Amputationen des Scheidentheiles, die von den verschiedensten Berliner Gynäkologen ausgeführt waren, und in welchen sich im Anschlusse an die Operation eine Para- bezw. Perimetritis posterior entwickelt hatte. Dieselbe entsteht nach der Ansicht des Vortragenden dadurch, dass die Nahtfäden im retrouterinen Bindegewebe liegen oder sogar das Bauchfell mitfassen. Dies ist auch der Fall, wenn die Absetzung der Portio vaginalis unterhalb des Niveau des hinteren Scheidengewölbes stattfindet, und wird bedingt durch eine eigenthümliche Umformung der Schnittwunde an der hinteren Lippe. Das Bindegewebe der Scheidenwand geht nämlich in zwei Bündeln, einem unteren und einem oberen, auf die hintere Lippe über, wodurch diese in zwei Abschnitte zerfällt, einen vorderen, dem Cervicalcanale angrenzenden, der in seinem festen Baue und dem Reichthume an Muskelfasern dem eigentlichen Gebärmuttergewebe entspricht, und einen hinteren, der unmittelbar eine Fortsetzung der Scheidenwand in die Gebärmutter hinein darstellt. Besonders bei Fällen von chronischer Metritis tritt dieses Verhalten scharf hervor. Führt man nun bei der Amputation den Schnitt über die hintere Fläche der Portio vaginalis, so trennt man das untere Bindegewebsbündel von dem vorderen Abschnitte ab, infolgedessen sich dieses mitsammt der Schleimhaut der Portio auf die Scheidenwand hin zurückzieht und mit dieser völlig verschmilzt. Die Zurückziehung des unteren Bindegewebsbündels bringt es ferner mit sich, dass das obere stark ausgezerrt und verdünnt wird. Infolgedessen liegt auch bei tiefer Absetzung bezw. keilförmiger Ausschneidang das retrouterine Bindegewebe und das Bauchfell dicht unter dem Grunde der Wunde. Pflanzt sich nun eine Entzündung der Stichcanäle in die Tiefe fort und Gelegenheit zu solchen Entzündungen ist durch die im Geschlechtsschlauche vorhandenen Mikroorganismen gegeben -, so ist das direct eine Para- bezw. eine Perimetritis posterior.

Um diesen Folgezustand zu vermeiden, der viel schlimmer ist als die Beschwerden bei chronischer Metritis, wegen deren so häufig die Absetzung gemacht wird, hält der Vortragende es für wichtig, die Wunde der hinteren Lippe zunächst durch versenkte Catgutnähte zu schliessen und darüber die Schleimhaut der Cervix und Vagina zu vereinigen. Er selbst verfuhr in zwei Fällen in der Weise, dass er nach bilateraler Spaltung, Ausschneidung und 
Vernähung der vorderen und Ausschneidung der hinteren Lippe die seitlichen Schnittenden durch versenkte Catgutnähte nach hinten und nach der Mitte hin zusammenzog. Hierdurch entstand eine sagittale, hinter dem Muttermunde liegende Wunde, welche zunächst durch eine versenkte und sodann durch eine oberflächliche Catgutnaht vereinigt wurde. Die Portio vaginalis gewann hierdurch von vornherein ihre natürliche zapfenförmige Gestalt. In beiden Fällen war die Operation angezeigt durch eine ausgedehnte folliculäre Erosion bei Metritis chronica. Bei letzterer allein zieht der Vortragende das Ausschaben mit nachfolgenden Einspritzungen von Chlorzink, bei Rissen der Cervix dasselbe Verfahren, in Verbindung mit der Emmet'schen oder einer entsprechenden Operation, der Absetzung der Portio vaginalis vor. Auch bei Stenosen ist die Amputatio portionis vaginalis entbehrlich. Bei der Stenose des äusseren Muttermundes allein genügt die Spaltung mit der Sims-Hegar'schen Umsäumung, der Vernähung der Schleimhaut der Cervix mit der Portio. Durch dieses Verfahren hat Gusserow in zahlreichen Fällen von langdauernder Sterilität Schwangerschaft erzielt. Bei der Stenose des ganzen Cervicalcanales bez. des inneren Muttermundes ist die Erweiterung angezeigt, die man am schnellsten und schmerzlosesten durch das A postoli'sche Verfahren erreicht. Hierdurch sah der Vortragende nach zwei Sitzungen Schwangerschaft eintreten bei einer 45jährigen Frau, die nur einmal im Beginne ihrer 20jährigen Ehe abortirt hatte.

\section{Besprechung.}

Martin (Berlin) hat die von dem Vorredner erwähnten üblen Folgezustände öfters beobachtet und durch verschiedene Abänderungen zu umgehen gesucht. Er stellt mit Genugthuung fest, dass der Vortragende seinen Standpunkt theilt.

Simpson (Edinburgh): Verengerung des Afters ohne Entfernung von Geweben. Simpson hat bei Prolapsus recti das Verfahren der Lappenspaltung mehrfach mit Erfolg angewendet. Ex umschnitt den After in seiner vorderen Hälfte und vernähte die so entstandene Wunde quer mit Silkworm. Hierdurch wird ohne Entfernung von Geweben nicht nur der After verengert, sondern auch der Aftercanal wieder hergestellt und ihm die regelmässige Richtung zurückgegeben. Die Lappenspaltung wendet Simps on nicht nur bei Perineoplastik, sondern auch bei BlasenArchir f. Gynäkologie. Bd. XXXIX. Hft. 1. 


\section{Verhandlungen der gynäkologischen Section d. X. internationalen}

scheidenfisteln seit 13 Jahren an. Bei Rissen der Cervix hatte er mit dem Verfahren keinen Erfolg.

Sinclair (Manchester): Ueber 100 Fälle von Emmet'scher Operation (Empfehlung der Operation bei tiefen Rissen der Cervix, welche Beschwerden machen).

Marcy (Boston): Plastische Operation am Beckenboden. Der lange Vortrag Marcy's, der eine der Tait'schen analoge Operation beschreibt, gipfelt in der Angabe, dass er schon fünf Jahre vor $\mathrm{W}$ erth die animalische Ligatur angewendet hat.

Edebohls (New York): Ein neues Nahtrerfahren bei Perineorrhaphie.

Balandin (Petersburg): Ein neuer Operationssaal, insbesondere für Laparatomien. In demselben sind die Plätze für 100 Zuschauer fast gerade über dem Operationstische angebracht, dabei aber durch Glaswände gänzlich von dem Operationsraume getrennt.

Derselbe: Operationsverfahren bei Dehiscenz der Narben nach Laparatomie. Die Haut wurde zu beiden Seiten des Bruches bis in die Muskeln hinein gespalten, und es wurden drei Reihen Nähte angelegt, welche die beiden Wundflächen über die Hernie hinweg vereinigten. Der Erfolg war ein befriedigender.

Truzzi (Mailand): Zwei Fälle von Castration wegen Osteomalakie. In dem einen handelte es sich um nicht puerperale Osteomalakie. Auf Grund seiner beiden Fälle sieht Truz zi in der Castration ein empirisches, aber recht sicheres Mittel für die Besserung und selbst Heilung der Osteomalakie.

Fehling (Basel): Wesen und Behandlung der puerperalen Osteomalakie. Die Erfolge der Castration bei der Osteomalakie sieht Fehling in dem Wegfalle der Ovulation. Die Osteomalakie verschlimmert sich zu jeder Menstruationszeit, - die Schmerzen nehmen sofort nach der Operation ab. Die entfernten Eierstöcke und Eileiter waren in allen Fällen ausserordentlich gefässreich. Die befallenen Frauen waren stets sehr fruchtbar. Diese sind nicht erkrankt, weil sie so viele Kinder geboren hatten, sondern meist begann die Krankheit früh, und trotzdem wurden noch zahlreiche Kinder geboren. Es drängt sich daher die Anschauung auf, dass die Krankheit eine erhöhte Fruchtbarkeit bedingt. Das Wesen der Krankheit liegt nach 
Febling in einer krankliaft erhöhten Thätigkeit der Eierstöcke mit reflectorischer Erregung der Vasodilatatoren der Knochen. Diese erzeugt eine Stauungshyperämie, welche zu einer erhöhten Resorption des ausgebildeten Knochens führt. Durch die Castration fällt diese reflectorische Wirkung auf die Dilatatoren fort. Die Osteomalakie ist daher eine Angioneurose der Knochen, eingeleitet durch trophische Störung der Eierstocksnerven. Sie zeigt eine gewisso Aehnlichkeit mit Struma oder mit dem Morbus Basedowii. Wenn die Entstehungsweise auch immer noch dunkel bleibt, so besitzen wir doch jedenfalls einen grossen Heilfactor in dem Kaiserschnitte nach Porro und der Castration.

Besprechung.

Winckel (München) bringt die verminderte Alkalescenz des Blutes mit der Osteomalakie in Verbindung. Eine Hyperämie der Anhänge bei der Castration hat er nicht gefunden.

Löhlein (Giessen) empfiehlt die von Kehrer zuerst angewandten warmen Soolbäder gegen Osteomalakie.

Fehling erwidert auf eine Frage Marocco's, dass die galvanische Behandlung der Osteomalakie möglicherweise Erfolg haben könne.

\section{Sitzung am 7. Angust Mittags.}

Vorsitzender: Lusk (New York).

Pawlik (Prag): Ueber einen Fall von Exstirpation der Blase. Bei der 57jährigen Kranken war ein Blasenpolyp, welchen Pawlik von der Vagina aus entfernt hatte, nach neun Monaten wiedergekehrt, und die bösartige Wucherung hatte sich über die ganze Blase verbreitet. Es wurden zunächst beide Harnleiter nach ihrer Sondirung freipräparirt, gespalten und ihre Schleimhaut mit der Scheidenschleimhaut vernäht. Für den Abfluss des Urins aus den Harnleitern wurde durch sorgfältiges Katheterisiren gesorgt. Die Einheilung der Harnleiter gelang vollständig. Als zweiter Act wurde die Sectio alta nach voraufgegangener Katheterisation der Harnleiter ausgeführt und die mit Emulsion aus Jodoform gefüllte Blase grösstentheils stumpf abgelöst. Die ziemlich starke Blutung wurde durch Tamponade gestillt. Nunmehr wurde die Scheidenwand dicht über dem Harnröhrenwulste quer gespalten, die Blase vorgezogen und am Orificium internum urethrae abgeschnitten. Dieses wurde alsdann 
theils mit der Scheidenwunde verbunden, theils nebst einer circulären Anfrischung des Scheideneinganges zu einer queren Vereinigung der Scheide benutzt. Vor dem Schlusse der Scheidenpähte wurden in beide Harnleiter elastische Katheter eingelegt und ihre Enden durch die Harnröhre herausgeführt. Die Bauchwunde wurde vernäht bis auf den unteren Theil. Die Verschliessung der Scheide heilte bis auf eine ganz kleine Fistel. Durch Zusammenziehung der Dammmuskeln kann die Kranke den Urin freiwillig entleeren. Beim Liegen wird er längere Zeit zurückgehalten.

\section{Allgemeines Thema: Künstliche Frühgeburt.}

Referent Parvin (Philadelphia): Ueber die Anzeigen der künstlichen Fr ühgeburt mit Rücksicht auf die Mutter. Als solche sind zu nennen: 1) Unstillbares Erbrechen. Von $10 \mathrm{ihm}$ aus der Literatur zugänglichen Fällen wurde $8 \mathrm{Mal}$ die Mutter geheilt, 5 Kinder lebend geboren. Die Todesfälle wären zu vermeiden gewesen. 2) Nierenkrankheiten. 3) Herzkrankheiten. 4) Lungenkrankheiten (capilläre Bronchitis, Pneumonia, Oedema, Phthisis. Von 10 Müttern kamen 6 mit dem Leben davon. 5) Nervenkrankheiten: Eklampsia, Meningitis. Von 9 Müttern genasen 6. Von 10 Kindern kam 1 todt zur Welt. 6) Acute Infectionskrankheiten. 7) Als häufigste Ursache: Abnormitäten des Beckens. Unter 988 Fällen von künstlicher Frühgeburt war eine solche $870 \mathrm{Mal}$ vorhanden.

Correferent Macan (Dublin) führt aus, dass die Gefahren der künstlichen Frühgeburt durch die Antisepsis sehr herabgemindert seien und bald ganz verschwinden werden. Schwierigkeiten erwachsen in den Fällen, wo die Interessen von Mutter und Kind entgegengesetzte sind, wie z. B. bei engem Becken. Je heilsamer hier die frühe Operation für die Mutter ist, desto gefährlicher ist sie für das Kind. Was den Kaiserschnitt betrifft, so ist der Mutter die Sachlage genau auseinanderzusetzen, und es sind ihr die verschiedenen Entbindungsverfahren, sowie deren Gefahren durchaus klar zu machen. Was die Methoden anlangt, so sind alle solche zu vermeiden, welche Blutungen setzen, weil das Blut bei längerer Dauer der Geburt sich zersetzen und so zu secundärer Infection führen kann. Die beste Methode scheint dem Redner die Einführung von Bougies und weiterhin die von Barnes'schen Blasen. 
Dobronravow (Kiew): Ueber die künstliche Frühgeburt. Empfiehlt die Erweiterung der Cervix durch Quellmittel.

Correferent Calderini (Parma) stellt folgende Thesen auf: 1) Im Interesse des Kindes muss man bei rhachitischem Becken die künstliche Frühgeburt aufgeben, wenn die Conjugata vera weniger als $7,5 \mathrm{~cm}$ beträgt. 2) Bei verengtem, nicht rhachitischem Becken gestatten die antiseptischen Vorsichtsmaassregeln die künstliche Frühgeburt einzuleiten bei einer Conjugata vera bis zu 8,5 und in gewissen Fällen noch mehr Centimetern. 3) Die Sterblichkeit der lebend geborenen Kinder bei künstlicher Frühgeburt kann durch sorgfältige Maassnahmen vermindert werden. 4) Die antiseptischen Vorsichtsmaassregeln haben in vielen Krankheitsfällen, welche die Schwangerschaft verwickeln, aus der künstlichen Frühgeburt eine bedeutsame Heilquelle gemacht. 5) Die beste Methode zur Einleitung der künstlichen Frïhgeburt wegen verengten Beckens sind heisse, mit dem gewöhnlichen Irrigator mittels des Fergusson'schen Speculum vorgenommene Douchen und die Einführung einer Bougie bis zum Fundus ateri. Bei Krankheiten ist es oft rathsam, den Eihautstich mit den oben angegebenen Methoden zu verbinden. -

Caldexini giebt gleichzeitig eine vergleichende Tabelle über die verschiedenen in Italien üblichen Operationsmethoden bei verengtem Becken, aus der sich ergiebt, dass die Sterblichkeit der Mütter wächst von der Wendung zur künstlichen Frühgeburt, zur Symphysiotomie, zum Forceps, zur Perforation, zum Kaiserschnitte nach Porro und endlich dem nach Sänger. Die Sterblichkeit des Kindes im Augenblicke der Geburt wächst stufenweise von der Operation nach Sänger zur Operation nach Porro, zur Symphysiotomie, zum Forceps, zu der künstlichen Frühgeburt und der Wendung. Letztere ergiebt also die höchste Sterblichkeit der Kinder.

Correferent Dohrn (Königsberg) stellt die folgenden Thesen auf: Bei Schwangeren mit Beckenenge mittleren Grades (7-8 cm Conjugata vera) ist in der Regel die künstliche Frühgeburt als das geeignetste Verfahren anzusehen. Für derartige Fälle ist der Werth der künstlichen Frühgeburt auch durch die neuerdings gebesserten Ergebnisse des Kaiserschnittes und der Perforation nicht erschüttert worden.

Zur Begründung dieser Thesen führt Dohrn 271 künstliche Frühgeburten wegen Beckenenge aus antiseptischer Zeit an, die 
150 Verhandlungen der gynäkologischen Section d. X. internationalen

$163=60,1$ Proc. lebend erhaltene Kinder ergeben. Dagegen lieferten bei denselben Personen 171 rechtzeitige Niederkünfte nur $50=29,2$ Proc. lebend erhaltene Kinder. Allerdings betrug die Mortalität der Mütter bei 318 künstlichen Frühgeburten noch 5 Proc. (16 Fälle). Dieselbe wird unzweifelhaft noch heruntergehen. So hatte Ahlfeld unter 111 Frühgeburten bei engem Becken nur 1 Todesfall der Mutter. Die Mortalität bei der Perforation beträgt zudem 5,6 Proc. (215 Fälle - 12 Todesfälle). Selbst Leopold verlor unter 23 Kaiserschnittsfällen noch 8,6 Proc. der Mütter, rettete allerdings 87 Proc. der Kinder. Doch fällt das gegenüber 60 Proc. der Kinder, wie sie durch die künstliche Frühgeburt gerettet sind, kaum ins Gewicht. Die Todesfälle der Mütter drücken den Erfolg wieder herunter.

Besprechung.

Leopold (Dresden) hat 75 künstliche Frühgeburten mit 1 Todesfalle und 42 Kaiserschnitte mit 4 Todesfällen ausgeführt. Leopold hält die künstliche Frühgeburt für eine sehr segensreiche Operation, deren Ergebnisse sich noch günstiger gestalten würden, wenn wir noch grössere Fortschritte in der Erhaltung des kindlichen Lebens und in der Diagnose der Zeit der Schwangerschaft machen würden. Der Kaiserschuitt muss vorwiegend den klinischen oder den entsprechenden geburtshülflichen Anstalten vorbehalten bleiben.

Löhlein (Giessen) hebt hervor, dass durch die bäufigere Ausführung des Kaiserschnittes nicht die Zahl der künstlichen Frühgeburten, sondern der Perforationen abnehmen soll. Er führt einen Fall mit einer Conjugata vera von $7,3 \mathrm{~cm}$ an, in dem nach zwei Mal vorhergegangener Geburt von todten Kindern die Frau selbst den Kaiserschnitt gewünscht, er aber die künstliche Frühgeburt vorgenommen hatte, die ein lebendes Kind von $2400 \mathrm{~g}$ Gewicht ergab.

Säuger (Leipzig) betont Löhlein gegenüber, dass der Kaiserschnitt neben der künstlichen Frühgeburt doch in Betracht komme, besonders bei stärker verengtem Becken, wo die Ergebnisse der künstlichen Frühgeburt für die Kinder sehr schlechte sind. Ueberhaupt sind die Ergebnisse für die Kinder bei künstlicher Frühgeburt noch ebenso ungünstig wie früher. Die Sterblichkeit der Mütter bei dem Kaiserschnitte wird geringer werden. Sänger sind 100 Fälle von Kaiserschnitt mit nur 5 Proc. Sterb- 
lichkeit bekannt. Er protestirt gegen den Vorschlag Le opold's, den Kaiserschnitt nur auf die Klinik zu beschränken.

Fehling (Basel) sieht auf 60 künstliche Frühgeburten mit keinem Todesfalle und mit 80 Proc. lebend entlassenen Kindern zurück. - Die beim Kaiserschnitte aus der Klinik entlassenen Kinder haben dieselbe schlechte Aussicht weiter zu leben, wie uneheliche klinisch geborene überhaupt, von denen 70-80 Proc. im ersten Lebensjahre sterben. Fehling räth als die für den praktischen Arzt und für die Klinik einfachsten und ungefährlichsten Methoden an: Die Tamponade der Cervix mit Jodoformgaze und dem von ihm abgeänderten Kolpeurynter von Barnes; darauf die frühzeitige combinirte Wendung.

Balandin (Petersburg) erwähnt Fälle, wo die künstliche Frühgeburt erst wochenlang nach der Bougierung und der Blasensprengung zu Ende ging. Trotzdem blieben die 43 von ihm so behandelten Mütter am Leben, und das Wochenbett war völlig regelmässig. Die letzten 20 Fälle gaben 19 lebende Kinder. Die lange Dauer der künstlichen Frühgeburt beruht auf der Fernhaltung von Sepsis und auf der verschiedenen Reizbarkeit der Gebärmutter.

Leopold Meyer (Kopenhagen) verwendet sich für die Perforation in der Hoffnung, in dem betreffenden Falle später Gelegenheit zur künstlichen Frühgeburt zu haben. Dies ist zwar nur eine Hoffnung, aber beim Kaiserschnitte besteht auch nur eine solche, dass die Frau den Kaiserschnitt überleben wird.

Kocks (Bonn) empfiehlt die von ihm schon früher angegebenen Tampons, durch welche nicht nur die Cervix, sondern auch das Scheidengewölbe ausgedehnt wird. Hierdurch ist der Wehen erzeugende Reiz ein grösserer.

de Cortejarena (Madrid): Ueber die Pathogenese, Prophylaxe und Therapie des Puerperalfiebers. Cortejarena sieht in Erkältung eine Ursache des Puerperalfiebers, eine fernere in der traumatischen Einwirkung bei Operationen, Tamponade u. s. w. Um das Fieber zu vermeiden, legt er die Wöchnerin in das beste Zimmer, hütet sie vor Erkältung und warnt sie vor dem frühzeitigen Wäschewechsel; ferner giebt er Ergotin. Bei physiologischen Entbindungen spielt daher die Antisepsis in der Therapie von Cortejarena keine Rolle. Bei pathologischen Geburten vermeidet man das Puerperalfieber durch Vermeidung jeder Gewalt und durch Anwendung - von Kamillenausspülungen. 
152 Verhandlungen der gynäkologischen Section d. X. internationalen

Dührssen (Berlin) erklärt eine von ihm angegebene Abänderung des Auvard'schen Kranioklaster, welche geeignet ist, die Brauchbarkeit des ausgezeichneten Instrumentes nicht unwesentlich zu erhöhen. Wie J. Veit hat Dührssen das innere Blatt mit einer zweischneidigen Spitze versehen, wodurch die Perforation sehr erleichtert wird. Ausserdem hat Dührssen den Schluss sicherer gemacht, weil ein Aufspringen des Kranioklaster starke Verletzungen und Quetschungen der mütterlichen Weichtheile zur Folge haben würde.

\section{Sitzung am 7. August Nachmittags. \\ Vorsitzender: Chrobak (Wien).}

Winckel (München): Ueber die Darmbrüche am Boden des weiblichen Beckens. Nach einem geschichtlichen Ueberblicke über die Fälle von Hernia vaginalis labialis führt Winckel aus, dass die Eingangspforte für diese Hernien die Stelle bildet, wo die Fascia pelvis interna von dem sehnigen vorderen Ansatze des Levator ani auf den Musculus obturator übergreift. Die Therapie besteht in der Zurïckbringung und Zurückhaltung durch ein Pessar. Die Hernia vaginalis labialis möchte er Hernia subpubica nennen. An diese reiht sich an die Hernia vaginalis (anterior, lateralis, posterior). Die Austrittsstelle aller dieser Brüche ist der Introitus vaginae. Dann folgt die Hernia pararectalis oder subtransversalis oder sacrorectalis. Hier ist die Bruchpforte hinter dem Musculus transversus perinei zwischen Rectum, Os coccygis und Tuber ossis ischii gelegen. Von diesen perinealen Brüchen zählt Winckel 8 Fälle auf. Seinen Vortrag schliesst Winckel mit einem Falle, wo zu gleicher Zeit eine Hernia subpubica, eine Hernia vaginalis (anterior et posterior) und eine Hernia subtransversalis vorhanden war. Eine Laparatomie war in diesem Falle wegen Verwachsung des Darmes im Bruchsacke erfolglos. Durch Spaltung, Aussehneidung des Bruchsackes und Vernähung von der Scheide her wurde Heilung erzielt.

Assaky (Bukarest): Ueber extraperitoneale Hysteropexie. Assaky hat in einem Falle die Ventrofixatio ohne Incision des Abdomen und in vier Fällen ohne Incision des Peritoneum ausgeführt. In dem ersten Falle, wo die Gebärmutter nur mit einer Nadel befestigt war, trat Rückfall ein; die anderen sind geheilt. Bei Hochlagerung des Beckens lässt sich das Bauchfell 
vermeiden; ebenso sind Blasenverletzungen zu umgehen; denn beim Heraufdrängen der Gebärmutter von der Scheide lässt sich der Ansatz der Blase an die Gebärmutter erkennen.

Picqué (Paris): Ueber Extrauterinschwangerschaft. Pi cqu é spricht sich über die Behandlung der Extrauterinschwangerschaft als die einer bösartigen Neubildung aus.

Boisleux (Paris): Ein Fall von Extrauterinschwangerschaft im drititen Monate.

J. Veit (Berlin): Ueber Hämatokele und Hämatoma. Veit schlägt zuerst strenge Einhaltung der verschiedenen Namen für Blutergüsse in der Nähe der weiblichen Geschlechtstheile vor. Haemorrhagia intraperitonealis - die freie Blutung in die Bauchhöhle; Hämatokele - die abgekapselte Blutung in die Bauchhöhle; Hämatom - die Blutung in das Bindegewebe. Aus einer freien Blutung in die gesunde Bauchhöhle entsteht niemals eine Abkapselung - Hämatokele. Kommt die Blutung zum Stillstande, so wird das Blut aufgesogen; sonst fübrt die Blutung bei ihrer Andauer zum Tode, weil intraperitonealer Druck auf die blutende Stelle zur Blutstillung nicht genügt. Vorbedingung zur Entstehung einer Hämatokele ist Abkapselung ïber der blutenden Stelle oder langsam erfolgende Blutung (regelmässige Art der Blutung bei Eileitersschwangerschaft) in eine schon vorher mit mehr oder weniger zahlreichen Verwachsungen versehene Bauchhöhle. Beim Hämatoma erfolgt die Blutstillung durch den Druck des nach aussen mit Bauchfell bekleideten Bindegewebes. Die Diagnose auf freie Blutung in die Bauchhöhle gründet sich auf die allgemeinen Zeichen der Blutleere ohne jeden Befund objectiver Art in der Bauchhöhle, wenn man jede andere innere Blutung ausschliessen kann. Freies Blut in der Bauchhöhle kann weder flüssig noch geronnen gefühlt werden. Die differentielle Diagnose zwischen Hämatokele und Hämatoma ist meist leicht und in schwierigen Fällen erfahrungsgemäss unwichtig. Nothwendig bei beiden Erkrankungen ist der Befund einer Geschwulst. Therapeutisch ist Einheit schon vorhanden über die Grundsätze bei Hämatoma und Hämatokele. Bei freier Blutung in die Bauchhöhle, völlig negativem Untersuchungsbefunde und schweren Erscheinungen allgemeiner Art muss die Bauchhöhle eröffnet werden. Veit empfiehlt zur schnellen Uebersicht die Operation mit stark erhöhtem Becken und behufs vollständiger Sicherheit der Blutstillung (bei 
154 Verhandlungen der gynäkologischen Section d. X. internationalen

Herkunft der Blutung aus einem Eileiter) die Unterbindung derArteria uterina und der Arteria spermatica in ihrer Continuität.

Besprechung.

L. Landau (Berlin): Die Hämatokele kann auch bei freier Blutung in die Bauchhöhle in Fällen von Eileitersschwangerschaft mit Berstung des Eies oder einer anderen ektopischen Schwangerschaft entstehen. Neben den beiden von $V$ eit angegebenen Verlaufsarten der freien Blutung ist dies die dritte, die Landa u bei Gelegenheit der Laparatomie beobachtet hat. Das freie Blut kann gerinnen, wenn das Epithel des Bauchfelles abstirbt, daun bilden sich auch Pseudomembranen und Abkapselungen. Uebrigens lässt sich das freie Blut in der Bauchhöhle durch Percussion nachweisen.

Law s on Tait (Birmingham) stimmt den Ansichten ron Veit bei und führt die Diagnostik der Hämatokele und des Hämatoma weiter aus.

Martin (Berlin) betont mit Tait, dass die Hämatome selten zu Eingriffen zwingen.

Nöggerath (Wiesbaden): Ueber sechs Fälle kleiner und mittelgrosser Ovarialkystome, welche durch Anwendung des inducirten Stromes vollständig zum Schwinden gebracht wurden. Der Strom war von geringer Stärke, aber sehr langer Dauer (1/4-1 Stunde).

Marocco (Rom): Ein glücklich verlaufener Fall von Sublimatintoxication. Nach der wegen bestehender Syphilis erfolgten Injection von $6 \mathrm{cg}$ Sublimat trat drei Stunden später eine Chorea musculorum und weiterhin Polyurie, Speichelfluss, Leibschmerzen ein. Die Temperatur betrug nur 35,8. Die Therapie bestand in Morphiuminjectionen und Chloralklysmata.

Neugebauer d. J. (Warschau): Anatom ische Beobachtung einer utero-cervico-sacro-promontorialen Synechie und nachfolgender Verwachsungen am Vorberge. (Usur der Cervix infolge einer schweren Geburt bei plattem Becken. Aus der Verwachsung mit dem hinteren Peritonealblatte der Douglas'schen Tasche hatte sich ein knöcherner spitziger Stachel von $1 \mathrm{~cm}$ Länge gebildet.) 
10. Sitzung am 8. August Vormittags.

Vorsitzender: Fochier (Lyon).

Schaternikoff (Moskau) legt einen Apparat vor, der sowohl als Douche-, als auch als Waschapparat zu verwenden ist, und den sowohl Aerzte wie Hebammen stets in ihrer Tasche bei sich führen sollen. Ferner zeigt er einen in Kliniken gut zu verwendenden Waschtisch, der mit Behältern in. Verbindung gesetzt ist, in denen sich antiseptische Lösungen befinden.

Keppler (Venedig): Das Geschlechtsleben des Weibes $\mathrm{nach}$ der Castration. Unter 46 Fällen doppelseitiger Castration habe er 39 Heilungen und 7 Todesfälle an Septikämie erlebt. In 15 Fällen besitzt er ausführliche Nachrichten über das Befinden der Kranken nach der Operation. Niemals war wegen Psychose, sondern stets wegen Organérkrankungen castrirt worden, und zwar 5 Mal wegen Myomá uteri, in den übrigen Fällen wegen Salpingitis oder Oophoritis. Was die letztere Gruppe anbetrifft, so ist eine (typische) Menstruation nie hinterher aufgetreten. Die Conjugata ist bei der Mehrzahl kürzer geworden; ebenso die Gebärmutter. Die Scheide wurde enger, die Brüste flacher, der Warzenhof verlor seine Färbung. Stärkere Beleibtheit trat in keinem Falle auf. Der Geschlechtstrieb blieb durchweg erhalten; die Castration ist somit nicht als Ehehinderniss anzusehen. Vielmehr haben sich mehrere vorher jungfräuliche Kranke verheirathet und leben in durchaus glücklicher Ehe. Bei der ersteren Gruppe trat nicht sofort Menopause ein; aber die vorher übermässigen Blutungen wurden sparsamer. Mehrere Male traten Psychosen auf, welche jahrelang bestehen blieben. Die Geschwülste schrumpften stets bedeutend zusammen. Auffallend war, dass diese 5 Kranke, welche vorher alle leidenschaftlich den Beischlaf liebten, ausnahmslos den Geschlechtstrieb verloren haben.

Bouilly (Paris): Die entfernteren Folgen der Abtragung der Anhänge der Gebärmutter. Auch Bouilly hält die Ergebnisse der Castration für das fernere Geschlechtsleben des Weibes für ausgezeichnete, sobald die Anzeige zu dieser Operation durch die Erkrankung der Eileiter und Eierstöcke gegeben war. Bei gleichzeitiger Para- und Perimetritis sind die Erfolge meist weniger günstig. Die frühere Ansicht, dass die Castration einen herabstimmenden Einfluss auf das fernere Leben der Frau übt, ist - die obige Anzeige vorausgesetzt - durchaus falsch. 
Clarke (Cambridge): Ueber die Wichtigkeit der frühzeitigen Diagnose der Pyosalpinx als Ursache einer eitrigen Beckenentzündung. Die allermeisten Beckenabscesse sind Folgeerscheinungen einer Erkrankung der Eileiter. Da nun die Salpingitis, so lange der Vorgang noch nicht auf die Umgebung übergegriffen hat, in verhältnissmässig ungefährlicher Weise durch die Laparatomie zu heilen ist, so ist das frühzeitige Erkennen der Pyosalpinx von hohem Werthe. Sobald das Beckenbindegewebe ergriffen ist, sinken die Aussichten auf vollständige Heilung durch Laparatomie bedeutend. Deshalb muss die Frühdiagnose „Pyosalpinx“ um jeden Preis gestellt werden. Redner tritt warm für eine Probelaparatomie zu diesem Zwecke ein.

Leopold Landau (Berlin): Therapeutische Erfahrungen über Eileitersäcke. Eileitersäcke sind heutzutage unbedingt zahlreicher als früher und verdanken ihre Entstehung oft dem Uebermaasse der Kunsthülfe. Für Hydrosalpinx betrachtet Landau die luftkissenartige Lagerung und Federung der Gebärmutter als pathognomonisch. Für manche diagnostisch zweifelhaft bleibende Fälle empfiehlt der Vortragende die Punction. Das Vorhandensein eines Tubensackes macht an sich noch keine Laparatomie nothwendig. Es ist zu unterscheiden: I) Die Hydrosalpinx. Diese kann von selbst heilen oder durch richtige Lagerung der Gebärmutter, durch Massage oder durch kleinere chirurgische Eingriffe (Punction) zur Heilung kommen. Die Punction von der Scheide aus hat Landau in Hunderten von Fällen zur Anwendung gebracht. II) Die Pyosalpinx. Hier kommt therapeutisch in Betracht: 1) der Einschnitt von der Scheidenwand aus, welche Redner warm empfiehlt; 2) liegen die Eitersäcke den Bauchdecken an, so schneidet man sie besser von den Bauchdecken aus an, nachdem man die nicht angewachsene Geschwulst mit den Bauchdecken vernäht hat; 3) wenn die perisalpingitischen Erscheinungen in den Vordergrund treten, dann kommt nur die Entfernung der Eileiter durch die Laparatomie in Frage. Unter 52 Laparatomien wegen Pyo- und Hydrosalpinx hat Landau nur einen Todesfall an Ileus erlebt. Bei einigen der Castrirten zeigten sich nervöse und psychische Störungen. Diese beobachtete Landau besonders bei Hysterischen, die von anderer Seite castrirt waren.

Menge (Berlin): Salpingitis gonorrhoica. Menge hat 26 Fälle von Salpingitis in der Martin'schen Klinik auf ihren bakteriellen Inhalt geprüft und $2 \mathrm{Mal}$ den Streptococcus 
pyogenes, $1 \mathrm{Mal}$ den Staphylococcus albus gefunden und gezüchtet. 1 Mal fand er einen Diplococcus, der alle Merkmale des N eisser'schen Gonococcus aufwies. Menge hält die Möglichkeit einer gonorrhoischen Peritonitis aufrecht.

Besprechung.

Zwe ifel (Leipzig) giebt Menge Recht, dass die Gonococcen Peritonitis hervorrufen können. Er selbst hat in 71 Fällen $7 \mathrm{Mal}$ Gonococcen nachgewiesen. Das bisher verneinende Ergebniss der künstlichen Infection des Bauchfelles mit Gonococcen steht im Widerspruche mit der klinischen Erfahrung.

Abel (Berlin): Zur Anatomieder Eileitersschwangerschaft nebst Bemerkungen zur Entwicklung der mensch lichen Placenta. Bei der Eileitersschwangerschaft beginnt in der fünften Woche die Umwandlung des Endometrium in Decidua. Der ausserbalb dès Fruchtsackes gelegene Theil des Eileiters bleibt meist unverändert. Im Bereiche des Fruchtsackes bildet die Eileitersschleimhaut eine Decidua tubaria vera. Das Epithel der Chlorionzotten ist ein dreifaches, das äusserste ist das Endothel der erweiterten mütterlichen Capillaren. Die intervillösen Räume sind die stark erweiterten mütterlichen Capillaren, deren Wände von den Chorionzotten nicht durchbrochen werden.

Boldt (New York): Beitrag zur Kenntniss der normalen Gebärmutterschleimhaut. Boldt hat beobachtet, dass die Utriculardrüsen sowohl in Längen- wie in Querschnitten von stäbchen- oder spindelförmigen Gebilden umgeben waren. Auch das Stroma der Schleimhaut war von solchen Bildungen durchsetzt. Durch genaue Untersuchungen stellte Boldt fest, dass diese Gebilde glatte Muskelfasern sind, welche die Drüsen sowohl der Cervix wie des Corpus uteri in Form eines Gellechtes korbartig umgeben. Sie stehen in Verbindung mit den Muskelbündeln der Gebärmutterwand. Sie sind am mächtigsten in der Grenzzone zwischen Schleimhaut und Wand. Ihre physiologische Bedeutung kann nur eine die Absonderung befördernde sein.

Gottschalk (Berlin): Zur Entwicklungsgeschichte der menschlichen Placenta. Die Untersuchungen wurden angestellt an zwei von L. Landau der Lebenden entnommenen schwangeren Gebärmüttern aus dem Anfange des II. und III. Monates.

Fall 1 (II. Monat). Das Schleimhautepithel ist verloren gegangen. Die Verbindung zwischen Chorion und Zotten ist theil- 
weise schon ziemlich fest. Die Zotten verlieren, sobald sie in die Decidua cingedrungen sind, ihr anfangs verdicktes Epithel. Die Drüsen erweitern und strecken sich mehr. Ihre Wand wird von den eingewanderten Zotten sehr leicht durchbrochen. Die Gefässe sind erweitert. Während die Arterien stark geschlängelt sind, verlaufen die Venen gestreckt. Nach innen hin verschwindet das Gefässendothel der Arterien, so dass wirklich arterielle Cavernen entstehen, in welche die Zotten stellenweise schon eingedrungen sind. Theilweise ergiesst sich das Blut auch freimündend in die Drüsen, welche so in Bluträume verwandelt werden.

Fall 2 (IlI. Monat). Die Zotten durchsetzen die Serotina wie eine Neubildung. Es ist eine Gefässneubilduug eingetreten. Die Arterien, Venen und Drüsen der Placeita grenzen unmittelbar aneinander, gehen ineinander über und bilden schliesslich unregelmässig begrenzte Bluträume. Die Zwischenzottenräume sind nichts Anderes als die vereinigten und vergrösserten mütterlichen Blutund Drüsenräume.

Sperber erläutert einen Fall von fötaler Knochenmissbildung.

\section{Sitzung am 8. August Mittags.}

Vorsitzender: Priestley (London).

\section{Allgemeines Thema: Die Elektrolyse in der Gynä- kologie.}

Apostoli (Paris): Die Anwendung der Elektricität erstreckt sich auf Fälle von Endometritis und Metritis, auf Fibrome, periuterine Entzündungen, Exkrankungen der Anhänge, Amenorrhoe, Dysmenorrhoe und Blutungen. Zunächst vertheidigt Apastoli die von ihm angewendeten starken galvanischen Ströme. Dieselben haben infolge der Wärme, welche sie in durchströmten Geweben erzeugen, eine Beschleunigung des Blutumlaufes und damit erhöhtes Aufsaugungsvermögen zur Folge. Am positiven Pole bringen sie eine Zerstörung der Mikroben zu Wege, Sie wirken schneller und Rückfälle sind seltener. Wichtig ist die intrauterine Anwendung der Elektricität. Die therapeutische Wirkung der Elektricität beruht also auf der Wirkung der Wärme und der nachfolgenden circulatorischen Drainage, ferner auf der antiseptischen und auf der polaren und interpolaren Wirkung. Die vaginale Galvanopunctur wendet A postoli jetzt nur auf wenige Millimeter Tiefe an mit einem dünnen Trocart, der bis auf die Spitze isolirt 
ist. Bei 912 Kranken hat A postoli 11499 Mal die Elektricität angewendet. Drei von ihnen sind gestorben; davon eine infolge von zu tiefer Galvanopunctur, welche in die Bauchhöhle eindrang. In dem zweiten Falle handelte es sich um eine wahrscheinlich eitrige Salpingitis, in dem dritten um ein Ovarialkystom. 30 der Kranken sind später schwanger geworden. Die conservative Gynäkologie findet in dem Galvanismus ihre sicherste und wirksamste Waffe.

Cutter (New York): Die Elektrolyse der Myome. Dieselbe ist in Amerika theilweise vor Apostoli, theilweise nach der Methode von Apostoli eingeführt. In durchaus origineller Weise hat Cutter seine Methode ausgebildet und mit derselben völlige Heilung, Verkleinerung der Geschwulst, Beseitigung der Schmerzen und der Blutungen erzielt. Unter 50 Fällen, die Cutter tabellarisch auff ührt, wurden durch den Galvanismus völlig geheilt 11 Fibrome, das Wachsthum aufgehalten in 25 Fällen, gebessert 3 Fälle, nicht gebessert 7, gestorben sind 4 .

Zweifel (Leipzig): Von den zahlreichen Thesen Zweifel's heben wir nur die hervor, dass die hohen Ströme von 175-200 und mehr Milliampère sehr schmerzhaft sind. Allerdings gewöhnen sich die Kranken an die Schmerzen. Die Galvanopunctur widerräth $\mathrm{Zw}$ eifel. Die einfache intrauterine Anwendung ist in allen Fällen, wo acute Entzündungen fehlen, vollkommen ungefährlich. Die Behandlung ist eine rein symptomatische. Allerdings wurden die Myome kleiner; aber nach dem Aufhören der Behandlung wachsen sie wieder. Bei älteren Kranken scheint die Verkleinerung Bestand zu haben. Einklemmungserscheinungen gehen zurïck. Die Anode stillt die Blutung; die Kathode scheint sie - wenigstens anfangs - eher zu vermehren. Das subjective Befinden der Kranken wird in der Regel in auffallender Weise gebessert.

La Torre (Rom): Beitrag zur Wirkung der Elektricität auf die Uterusfibrome. La Torre beschreibt einen Fall, wo vor der Entfernung des Fibroms die elektrische Behandlung angewendet worden war. Die Geschwulst war in Erweichung und fettige Umwandlung übergegangen. Die Schleimhaut der Gebärmutter war hochgradig atrophirt.

Massin (Petersburg): Ueber die Ergebnisse der Elektrotherapie bei Fibroma uteri. Massin hat ein Verschwinden der Blutung als Regel, Verkleinerung der Geschwulst nie gefunden. Das Allgemeinbefinclen besserte sich stets auf- 
fallend. - Fälle von Oophoritis, die schon zur Castration bestimmt waren, wurden durch die Anwendung des constanten Stromes so gebessert, dass die Operation aufgegeben wurde. Die Wirkung des Stromes sieht Massin in der Aetzung und der Katalyse.

Salvat (Bordeaux) sah bei drei Kranken mit subacuter und chronischer Parametritis durch intrauterine positive Galvanokaustik, verbunden mit der Massage, die Aufsaugung der Exsudate rasch eintreten. Einfache Verwachsungen wurden durch die Elektricität nicht rascher beseitigt, als durch die Massage allein. Die begleitende Leukorrhoe verschwand rasch.

Danion (Paris) sieht in der elektrokaustischen. Methode bei Uterusmyomen einen ungeheuren wissenschaftlichen Irrthum. Die kaustische Wirkung betrifft nur den inneren Muttermund. Die Galvanopunctur soll nur oberflächlich ausgeführt werden und kann daher keine Wirkung haben. Die Apostoli'sche Methode weist eine Sterblichkeit von 5-6 Proc. auf. Die von Danion angegebene Methode des elektrischen Tampons mit Stromwendung erzielt viel raschere und vollständigere Erfolge ohne die geringste Gefahr, wie hundert Fälle des Vortragenden beweisen.

Goubaroff (Moskau) hat an Gebärmüttern, die wegen Carcinoma entfernt wurden und vorher mittels Elektricität behandelt waren, nachweisen können, dass der positive Pol eines Stromes von 170 Milliampère von fünf Minuten Dauer die Schleimhaut der Gebărmutter austrocknet und ätzt, während der negative $\mathrm{Pol}$ unter denselben Bedingungen bei einer Stärke von 150 Milliampère eine Erweichung der Schleimhaut herbeiführt. Bei interstitiellem und submukösem Myom ist die galvanokaustische Methode, bei dem subserösen die Galvanopunctur zu empfehlen. Die besten Erfolge giebt die Elektricität bei nicht entartetem und isolirtem Myom. Die Blutungen, Schmerzen und Einklemmungserscheinungen werden häufig beseitigt. Die Faradisation beseitigt die Schmerzen bei Entzündungen der Gebärmutter und der Anhänge. Bei Carcinoma kann man die Schmerzen durch einen Strom von 1000 M.-A. innerhalb fünf Minuten beseitigen. Unter 500 so behandelten Fällen ist niemals irgend ein Unfall aufgetreten. Bei sehr starkem Strome muss man hin und wieder Chloroform anwenden. Die Galvanopunctur bringt vereinzelte, nicht entartete Myome zur Verkleinerung bezw. zum Verschwinden, wie Goubaroff durch Laparatomie nachgewiesen hat. In einem Falle von Extrauterinschwangerschaft wurde durch 15 Galvanopuncturen eine völlige 
Auflösung der. Placenta und des sechsmonatigen Fötus bewirkt, was ebenfalls durch Laparatomie festgestellt wurde. Dasselbe kann bei Hämatokele und nicht eitrigen Exsudaten erzielt werden. 20 Fälle von Extrauterinschwangerschaft bis zum dritten Monate wurden durch die Galvanopunctur völlig geheilt. Der tägliche Gebrauch der bipolaren Faradisation in einer Dauer von 15 bis 30 Minuten hatte die besten Erfolge bei Pruritus, Ischurie, Dysurie, Vaginismus und Dyspareunia.

Mac Ginnis (New York) bestätigt ebenfalls die günstigen Erfolge der Apostoli'schen Methode bei den bezeichneten Erkrankungen.

Gautier vertheidigt auf Grund eigener Erfahrungen die Methode von Apostoli gegen die Angriffe von Danion.

Spanton (Hanley) berichtet über eine Anzahl von Fällen, bei denen die Anwendung der Elektricität von bestem Erfolge begleitet war. Ebenso

Engelmann (St. Louis).

Bröse (Berlin) hat bei interstitiellem und submukösem Myoma die Apostoli'sche Methode seit zwei Jahren in 16 Fällen angewendet. Von diesen sind mehrere seit $1 / 4-1^{1 / 2}$ Jahren geheilt. Die Gebärmutterhöhle verkleinerte sich in allen Fällen um 1 bis $2 \mathrm{~cm}$. Zehn Kranke wurden wegen Blutungen behandelt und von diesen befreit. Von den übrigen 6 Kranken mit grossem Myoma sind 4 von ihren Beschwerden geheilt; in den beiden anderen Fällen wurde eine bedeutende Verkleinerung. der Geschwulst erzielt. Die Anwendung der vaginalen Kugelelektrode verwirft Bröse. Je länger die Gebärmutterhöhle ist, desto schwerer sind die Blutungen zu heilen. Daher wird die galvanische Behandlung nie die Myotomie ersetzen können. Ausgezeichnet wirkt der galvanische Strom bei chronischen Beckenausschwitzungen. Diese werden oft, nachdem sie jedem anderen Behandlungsverfahren Trotz geboten haben, nach kurzer Zeit aufgesogen. Bröse wendet auch hierbei die Elektricität stets intrauterin an. Bei Verknüpfung mit Endometritis nimmt er die Anode, sonst die Kathode. Bedingung ist, dass alle acuten und subacuten Erscheinungen verschwunden sind. Bei Endometritis, die mit Entzündung in der Umgebung der Gebärmutter verknüpft ist, zieht Bröse den Galvanismus der chirurgischen Behandlung vor.

Leopold Meyer (Kopenhagen) betont den hohen Werth der A postoli'schen Behandlung der Fibromyome als symptomatisches Archiv f. Gynäkologie. Bd. XXXIX. Hft. 1. 
162 Verhandlungen der gynäkologischen Section d. X. internationalen

Behandlungsverfahren. Die Krankheit selbst wird zwar nicht geheilt, aber die Kranke wird von den lästigen Erscheinungen befreit. - Meyer hat nur geringe Schmerzhaftigkeit bei Anwendung selbst starker Ströme gesehen.

\section{Sitzung am 8. August Nachmittags.}

Vorsitzender: Parvin (Philadelphia), später Wyder (Zürich).

Murphy (Sunderland): Die Behandlung der Placenta praevia. Murphy empfiehlt bei jedem diagnosticirten Falle von Placenta praevia, die künstliche Geburt dadurch einzuleiten, dass die Placenta so weit wie möglich abgelöst und dann ein Barnes'scher Kolpeurynter eingelegt wird. Unter 42 so behandelten Fällen von Placenta praevia verlor Murphy 2 Mütter; 20 Kinder wurden lebend geboren.

Halbertsma (Utrecht): Die operative Behandlung bei Eklampsie. Halbertsma betont die grosse Sterblichkeit für Mutter und Kind bei vor der Geburt auftretender Eklampsie. Selbst bei diaphoretischem Behandlungsverfahren und bei Anwendung grosser Dosen von Morphium und Chloroform beträgt die Sterblichkeit in den von Stumpf zusammengestellten 20 Fälien für die Muitter 17 Proc., für die Kinder 77 Proc. Drei Franen starben unentbunden. $6 \mathrm{Mal}$ hat man, von diesen Erwägungen ausgehend, bei Eklampsie vor oder im Beginne der Geburt in Holland den Kaiserschnitt gemacht. Nur eine Frau und ein Kind starben. Allerdings wurde in diesem Falle die Operation im letzten Stadium ausgeführt. Der günstige Einfluss der Fntbindung durch den Kaiserschnitt auf die Convulsionen war sehr auffallend. Nur in einem von fünf Fällen traten nach der Operation noch drei leichte Anfälle auf. Die am meisten eingreifende Operation hat also, anstatt Krämpfe zu veranlassen, diesen ein Ende gemacht. Die Gefahr der Blutung bei während der Schwangerschaft ausgefübrtem Kaiserschnitte ist nicht sehr gross (und sie lässt sich durch die Tamponade nach dem Vorschlage des Referenten ganz beseitigen. Ref.). Von 10 Kaiserschnitten in der Schwangerschaft, die Halbertsma bekannt sind, nahmen 2 einen ungünstigen Verlauf (20 Proc.). Für die Fälle, in denen der supravaginale Theil der Cervix verstrichen ist, zieht Halbertsma dem Kaiserschnitte die tiefen Einschnitte in die Cervix nach dem Dührssen'schen Verfahren vor. Die Schlusssätze Halberts ma's 
lauten: Bei der Eklampsie am Ende der Schwangerschaft und im Beginne der Geburt stelle man öfters eine ungefährliche operative Therapie derjenigen mit narkotischen Mitteln und heissen Bädern zur Seite. In den ungünstigsten Fällen - z. B. bei' vollkommener Anurie - greife man auch zu gefährlicheren Operationen. Man lasse keine Frau unentbunden sterben.

Frank (Köln): Ueber die Behandlung des Stumpfes bei der Operation nach Porro. Frank berichtet über sechs Fälle, bei welchen er den Gebärmutterstumpf in die Scheide hinein umstülpte. Nur eine Kranke starb. - Das Verfahren verbindet die Sicherheit der extraperitonealen Behandlung des Stieles mit der schnellen Heilung und der Eleganz der intraperitonealen Behandlung. Nach zwei bis drei Wochen können die Operirten die Anstalt verlassen.

Chrobak (Wien) bemerkt bei der Besprechung, dass er diese Behandlung des Stumpfes schon vor 20 Jahren empfohlen habe, aber jetzt kein Anhänger derselben mehr sei.

Winter (Berlin): Zur Behandlung des Abortus. Winter hat in einer Reihe von Fällen Untersuchungen über das Schicksal der bei Aborten in der Gebärmutter zurückbleibenden Decidua angestellt. In 15 Fällen, in denen die ganze Decidua, der Gebärmutter fest anhaftend, zurückblieb, bildete sich die Gebärmutter zur Norm zurück. Die Menses kehrten in regelmässiger Weise nach vier Wochen wieder. In 4 Fällen trat Empfängniss ein und die Schwangerschaft verlief regelmässig. In 30 anderen Fällen wurden nur die hin und her flottirenden Fetzen entfernt. Der Lochialfluss dauterte danach - ebenso wie in der ersten Gruppe - acht Tage. Die Menstruation trat nach vier Wochen ein. Die erste war meist stark. In 12 von diesen 30 Fällen war innerhalb der ersten Monate Empfängniss eingetreten, davon $4 \mathrm{Mal}$ schon nach einem Monate. Daraus folgt, dass die vollständige oder theilweise Zurückhaltung der Decidua weder auf den Verlauf des Wochenbettes, noch auf die späteren Functionen einen ungünstigen Einfluss übt. Nachfolgende Blutung oder mangelhafte Rückbildung bei Aborten schiebt Winter auf das Zurückbleiben von Chorionzotten oder zum grössten Theile schon gelöster Fetzen der Decidua. Man soll daher bei gesunden Frauen die anhaftende Decidua vera in der Gebärmutter zurücklassen und nur die lose hin und her flottirenden Fetzen entfernen. Eine grundsätzliche Entfernung der Decidua vera hält 
164 Verhandlungen der gynäkologischen Section d, X. internationalen

Winter für falsch, weil sie unnöthig ist und zu dem gefährlichen Missbrauche der Cürette führt.

Fochier (Lyon): Ueber eine Achsenzugzange. Empfehlung und Beschreibung derselben.

Cameron (Toronto): Ueber Kaiserschnitt mit drei erfolgreichen Fällen. Cameron rerwirft die Schnürung mittels des Schlauches und legt nur tiefe Nähte an.

Auvard (Paris): Ueber die intrauterine Tamponade in der Geburtshülfe. Auvard giebt eine Uebersicht über die Entwicklung und die Erfolge der Dührssen'schen Tamponade der Gebärmutter bei Blutungen nach der Geburt auf Grund einer Statistik von 67 Fällen mit 6 Todesfällen. 3 davon starben an der vorher schon vorhandenen äussersten Blutleere, 1 an ebenfalls schon vorhanden gewesenem Oedema pulmonum (infolge von Eklampsie), 1 an Septichämie, 1 an Tuberkulose. Somit ist die Tamponade ein ungefährliches Verfahren, sicherer als alle anderen, und wirkt lebensrettend, wenn sie nicht zu spät angewendet wird. Sie wird ihren Platz in der geburtshülflichen Praxis finden.

Besprechung.

Fochier (Lyon) spart die intrauterine Tamponade für die Fälle von Placenta praevia auf, in denen die Zusammenziehung der Gebärmutter die Blutung nicht stillen kann. Damit die Tamponade keine Expulsivbewegung hervorrufe, drückt er den Tampon zwischen vier in die Scheide eingeführten Fingern und der anderen auf dem Fundus uteri liegenden Hand zusammen. Fochier hat dieses Verfahren mit Erfolg in zwei Fällen angewendet.

Pasquale (Rom) hat die Tamponade nach dem Dührssen'schen. Verfahren in zehn Fällen mit dem Erfolge angewendet, dass die Blutung sofort stand, und das Wochenbett regelmässig verlief. Daher muss dieses Verfahren sich einen dauernden Platz in der Behandlung der Blutungen erwerben wegen seiner grossen Sicherheit und der Einfachheit seiner Anwendung.

Dührsen (Berlin) dankt Herrn Auvard ganz besonders für seinen Vortrag, weil dieser der Erste gewesen ist, welcher mit Fraipont das Verfahren praktisch versucht hat, und Herrn Pasquale ist er besonders verpflichtet, weil er zu den vielen hervorragenden ausländischen Geburtshelfern gehört, die Dührssen auf dem Congresse ihre zahlreichen durch die Tamponade erzielten überraschenden Erfolge mitgetheilt haben. Die Tamponade hat 
bereits die Feuerprobe bestanden. Von mehr als 200 Fällen, in denen sie angewendet worden ist, wurde nur in 4 Fällen die Blutstillung nicht erreicht. In 1 Falle war, von falschen theoretischen Voraussetzungen ausgehend, nur locker tamponirt worden (von Velits); in den 3 anderen Fällen handelte es sich um Placenta praevia, bei denen nur. mit Gaze tamponirt war, obgleich Dührssen schon von vornherein hervorgehoben hatte, dass man bei Blutungen aus dem unteren Gebärmutterabschnitte den unteren Theil der Scheide noch mit der weniger durchlässigen Watte ausstopfen solle. Sollte es trotzdem noch nach aussen bluten, so würde Dührssen den ganzen Gebärmutter-Scheidencanal mit trockener Watte ausfüllen. Wenn es einmal nach sorgfältiger Tamponade nach innen bluten und die Gebärmutter sich ausdehnen sollte, so würde man dem durch Andrücken der Gebärmutter gegen den innen liegenden Tampon zu begegnen haben. Auf diese Weise lassen sich nach Dührssen alle atonischen und zahlreiche Rissblutungen beseitigen - ob auf physiologische oder nicht physiologische Weise, das erscheint Dührssen ganz gleichgültig; denn, wie schon $K$ üstner bemerkte, heiligt nur der Erfolg das Mittel! Gegenüber dem absprechenden Urtheile von 0lshausen auf dem Freiburger Congresse möchte $\mathrm{D}$ ührssen ausdrücklich feststellen, dass für Deutschland und auch für das Ausland bereits die Zeit gekommen ist, wo hervorragende Kliniker und zahlreiche Praktiker in der Tamponade des Gebärmutter - Scheidencanales ein ungefährliches, nützliches und in manchen Fällen allein wirksames Mittel bei atonischen und zahlreichen Rissblutungen sehen. Möge die heutige Verhandlung - so schliesst Dührs sen - dazu beitragen, dạss die Tamponade des Gebärmutter-Scheidencanales auch in England und Amerika Eingang finde. Möge die Zeit bald kommen, wo in der Geburtshülfe, ebenso wie in der Chirurgie, dem Liquor ferri das Todesurtheil gesprochen wird. Möge endlich das jüngst empfohlene Mittel bei atonischen Blutungen - die künstliche Umstülpung und Schnürung der umgestülpten Gebärmutter - nie zur Anwendung kommen. Die Frauen, welche man auf diese Weise vor Verblutung gerettet hat, würden dann am Shoc zu Grunde gehen! Im Anschlusse an seine Bemerkungen erläutert Dührssen eine von Dr. Vowinckel angegebene Büchse, in welcher die hydrophile gaze, deren sich Dührssen jetzt fast ausschliesslich bedient, sterilisirt und welche dann direct in die Praxis mitgenommen wird. 
v. Ramdohr (New York) hat in der New Yorker medicinischen Monatsschrift bereits die Aufmerksamkeit der dortigen Aerzte durch Veröffentlichung einiger glïcklich verlaufener Fälle auf das Dührssen'sche Verfahren gelenkt und in weiteren vier Fällen Gelegenheit gehabt, es kennen und schätzen zu lernen. Nur eine Kranke ging an Eklampsie zu Grunde; Sepsis bekam keine. Die Blutung stand rasch in allen Fällen, nachdem er alle üblichen Mittel (ausser Liquor ferri) erfolglos angewendet hatte. Er weiss, dass auch mehrere seiner Fachgenossen das Verfahren mit bestem Erfolge angewendet haben und von der neuen Therapie entzuickt sind. Ramdohr wird gegebenen Falles heute noch viel rascher zu dem Mittel greifen, als früher.

Vulliet (Genf) zeigt Photogramme und Abgüsse des Inneren der Gebärmutter, die er nach Erweiterung der Cervix nach seiner bekannten Methode gewonnen hat, und schildert die Vorzüge derselben in diagnostischer und therapeutischer Beziehung (bei Endometritis, Myomata intramuralia, Carcinoma inoperabile, Retroflexiones, Haemorrhagiae). Gegen die Dührssen'sche Methode wendet er die Gefahr der Jodoformintoxication ein.

Kocks (Bonn) entgegnet darauf, dass D ührssen neuerdings nur aseptische Gaze verwendet. Weiterhin vertheidigt Kocks das von ihm vorgeschlagene Verfahren der Umstülpung gegen den Angriff von Dührssen. Denn die Fälle von chronischer Umstülpung thun dar, dass solche Kranke durchaus nicht an Shoc gestorben sind. Indessen will Kocks sein Verfahren doch nur auf solche Fälle beschränken, in denen dem Arzte die Dührssen' sche Gazebüchse und die lange Pincette nicht zur Verfügung stehen.

Landau (Berlin) rühmt die Erfolge des Vulliet'schen Erweiterungsverfahrens.

\section{Sitzung am 9. August Vormittags.}

Vorsitzender: Winckel (München).

Engström (Helsingfors): Zur Aetiologie des Myoma uteri. Engström tritt dafür ein, dass die von der Zeit der Pubertät bis zur Menopause vor sich gehende Hyperämie der Gebärmutter die Entwicklung der Myome begünstige, ja vielleicht hervorrufe. Weiterhin führt Engström vier Fälle an, in denen Mutter und Töchter bezw. Schwostern an Myomen erkrankt waren 
- Beobachtungen, die eine embryonale Anlage der Myóme wahrscheinlich machen.

Cutter (New York): Ernährung als Therapie bei Fibroma u teri. Möglichst günstige hygienische Bedingungen üben einen vortheilhaften Einfluss auf die Rückbildung der Myome aus. Als Unterstützungsmittel sind Massage, Galvanismus und psychische Einflüsse zu betrachten.

Eastman (Indianopolis): Ein Operationsverfahren zur Entfernung von Fibromata uteri. In zehn Fällen hat Eastman mit Hülfe eines besonderen Instrumentes das Scheidengewölbe breit von der Bauchhöhle aus geöffnet, die Unterbindungsfäden zur Scheide herausgeleitet und von der Scheide aus ein Drainrohr eingelegt. In den meisten Fällen wurde die ganze Gebärmutter weggenommen.

Fritsch (Breslau): Ueber Myomoperationen. Fritsch ist grundsätzlich für eine ausgedehntere Indicationsstellung der Myomotomie. Gegenüber der immer mehr sinkenden Lebensgefahr der Operation steht die völlige Sicherheit der Heilung. Die Castration lässt Frits ch nur bei grosser Schwäche und bei solchen Geschwülsten gelten, die noch von der Scheide aus zu entfernen sind, die Ausschälung nur bei breitbasig aufsitzenden subperitonealen $\mathrm{Po}_{0}$ lypen. Bei intraligamentären Myomen, wo eine Abbindung der Ligamenta lata nicht möglich ist, näht Fritsch den Sack in die Bauchwand ein und stillt die Blutung durch Tamponade - oder tamponirt den Sack, schliesst ihn durch eine. Tabaksbeutelschnurnaht und legt von der Scheide eine Gegenöffnung an. In beiden Fällen muss die Scheide fest ausgestopft werden. Von 60 nach . seiner (extraperitonealen) Methode operirten Fällen hat Fritsch 8 verloren. Für die Methode der Zukunft hält Fritsch die Totalexstirpation.

Besprechung.

Lawson Tait (Birmingham) rühmt die durch Castration bei Myomen von ihm erzielten Erfolge (219 Fälle, 4 Todesfälle= 1,8 Proc.).

Martin (Berlin) vertritt die Ausschälung bei Myoma uteri, nach welcher $8 \mathrm{Mal}$ Gravidität beobachtet wurde, und bemerkt Tait gegenüber, dass nach der Castration das Aufhören der Menstruation nicht in allen Fällon festgestellt wurde.

Nagel (Berlin): Erklärung einiger Glasphotogramme, welche verschiedene Entwicklungsstufen der Gebärmutter und der 
Scheide darstellen. An diesen Präparaten sieht man, dass von vornherein der Geschlechtsstrang eine seichte, dorso-ventrale Krümmung mit vorderer Concavität hat. Die Lage der inneren weiblichen Geschlechtstheile mit nach vorn über die Fläche geneigter Gebärmutter ist somit die ursprüngliche. Es besteht ferner von vornherein ein Unterschied in der Epithelbekleidung des proximalen und des distalen Abschnittes des Geschlechtsstranges. Aus dem ersteren bildet sich die Gebärmutter, aus dem letzteren die Scheide.

Edebohls (New York): Zwei Fälle von peritonealer Hämatokele von ungewöhnlicher Ausdehnung. Behandlung durch Abdominalsection. Heilung.

Derselbe: Abänderung der Alexander Adam'schen Operation. Die Aenderung besteht darin, dass Edebohls den ganzen Leistenring spaltet und das Ligament fasst. Dreiviertel des Ligamentes werden dann herausgezogen. Unter 26 auf diese Weise herausgezogenen Ligamenten riss keins. Das Ligament wird dann vernäht und der überschüssige Theil weggeschnitten. Mit dieser Naht wird zu gleicher Zeit der geöffnete Leistencanal geschlossen. Die Drainage wird durch Silkwormgut bewirkt. In 4 Fällen handelte es sich um Prolapsus, in den anderen um Retroflexio uteri.

Boisleax (Paris): Ueber Myomotomie. Boileux fand bei 10 von Martin entfernten myomatösen Vteri 4 Mal im Fundus uteri Keime, darunter $2 \mathrm{Mal}$ pathogene; $7 \mathrm{Mal}$ fanden sich im Collum Keime. Daher muss die Operation streng antiseptisch ausgeführt und der Stumpf cauterisirt werden.

Raineri (Vercelli): Beitrag zur Lehre der Placenta praevia. Raineri betont die Bedeutung der regelwidrigen Grösse der Placenta für die Aetiologie der Placenta praevia und fordert daher auf, weiterhin auf diesen Punkt zu achten.

Arendt (Berlin): Die Zusammenziehungsfähigkeit der Gebärmutter und ihre praktische Verwerthung. Arendt hat schon bei blosser Untersuchung, noch viel mehr aber bei der Massage Zusammenziehungen der Gebärmutter eintreten sehen. Dasselbe wird auch durch andere Handgriffe erreicht, besonders durch eine Verschiebung der Gebärmutter nach hinten oben rechts oder links. Hält man die Gebärmutter in dieser Lage etwa 15 Secunden fest und lässt sie dann in ihre normale Lage zurückgehen, so wird man regelmässig eine Veränderung der Gebär- 
mutter in Form, Grösse und Festigkeit wahrnehmen. Daher der Erfolg dieser Behandlung bei Metritis chronica, Endometritis und Hyperaemia uteri. Die Zusammenziehung der schwangeren Gebärmutter ist viel schwächer. Durch Massage des Isthmus zieht sich derselbe bei Retroflexio uteri zusammen. Das Corpus hebt sich und die Gebärmutter kann leicht zurückgebracht werden.

Poussié (Paris): Erläuterung von Tafeln über den Geburtsmechanismus.

Meyer (Tübingen): Zur Therapie der Geburt in Vorderhauptslage. In drei Fällen gelang es Meyer, durch äussere und innere Handgriffe die kleine Fontanelle nach vorn $\mathrm{zu}$ bringen.

v. Dombrowsliy (Petersburg): Eine Dermoidcyste mit eigenthümlicher Knochenbildung. Es fand sich in der Dermoidcyste ein dem Becken ähnliches Gebilde.

Pozzi (Paris): Ein neues Verfahren zur Einleitung der künstlichen Frühgeburt. Pozzi hat zu diesem Zwecke ein Dilatations-Instrument mit drei Armen angegeben, durch welches sich in kurzer Zeit eine Erweiterung bis zu $10 \mathrm{~cm}$ erzielen lässt.

La Torre (Rom): Vorzeigung eines Instrumentes zur Erweiterung der verengten Scheide.

Mackenrodt (Berlin): Demonstration von Präparaten aus der Martin'schen Klinik. (Nachträglich eingereicht.)

Das eine Präparat stellt eine Combination eines grossen Orarialkystoms mit zwei faustgrossen Dermoiden dar. Bei der Laparatomie Verunreinigung der Bauchhöhle durch Dermoidinhalt. Tod an Ileus. Mehrere andere Präparate zeigen nichtpuerperale Ovarialabscesse. Die Ursachen derselben lagen in intrauterinem Katarrh bezw. Behandlung. Die Infection erfolgte wahrscheinlich auf lymphatischem Wege, die auch bei den analogen Tubenerkrankungen eine Rolle spielt. Bei Platzen von Eitersäcken reinigt A. Martin nachher das Peritoneum mit einem mit ausgekochtem Olivenöl getränkten Schwamme.

Wincke I (München): Schlussansprache.

Winckel berichtet, dass sich 450 Mitglieder in die Sectionsliste eingetragen haben. Die Zahl der Vorträge betrug 118, die Zahl der an den Besprechungen theilnehmenden Redner 51. 